\title{
Unfree gauge symmetry in the BV formalism
}

\author{
D. S. Kaparulin ${ }^{\text {a }}$, S. L. Lyakhovich \\ Department of Quantum Field Theory, Tomsk State University, Lenin ave. 36, Tomsk 634050, Russia
}

Received: 2 August 2019 / Accepted: 17 August 2019 / Published online: 27 August 2019

(C) The Author(s) 2019

\begin{abstract}
The BV formalism is proposed for the theories where the gauge symmetry parameters are unfree, being constrained by differential equations.
\end{abstract}

\section{Introduction}

The Batalin-Vilkovisky (BV) formalism ${ }^{1}$ was initially proposed [1-4] as a tool for quantizing classical gauge field theories. Later on, the scope of applications of the formalism has been extended to a large variety of problems in physics and mathematics ranging from consistent inclusion of interactions in gauge field theories to the characteristic classes of various manifolds.

Given the Lagrangian, the BV-BRST embedding of the theory is a well-known straightforward procedure [5] if certain regularity conditions are obeyed by the original field equations and their gauge symmetry. These regularity conditions are also generalized for not necessarily Lagrangian field equations to provide their BV-BRST embedding [6,7]. We mention two conditions which are assumed to hold true for the original field theory to admit the usual BV-BRST embedding: (i) the gauge symmetry parameters are arbitrary functions of space-time coordinates, i.e. they are not constrained by any equations; (ii) any on-shell vanishing function of the fields and their derivatives can be spanned by the left hand sides of the field equations and their differential consequences. These two assumptions, being critical for the BV formalism construction, are violated in some field theory models of a current interest. Examples are given in Sect. 6. As one can see from the examples, both the assumptions are usually violated simultaneously. Once the gauge transformation parameters are constrained by equations, the gauge symmetry is named unfree.

\footnotetext{
${ }^{1}$ Also known as BRST (Becci-Ruet-Stora-Tutin) field-anti-field formalism.

a e-mail: dsc@phys.tsu.ru
}

In the recent article [8], the defining relations are found of the unfree gauge symmetry algebra. The algebra of gauge symmetry with unconstrained gauge parameters is constructed starting from two basic ingredients: the action functional and gauge symmetry generators. In the unfree case, two more basic ingredients are involved: the operators of gauge parameter constraints and the mass shell completion functions. The first extra ingredient defines the equations constraining gauge symmetry parameters. The completion functions constitute the generating set of the on-shell vanishing quantities such that do not reduce to the left hand sides of the Lagrangian equations and their differential consequences. Proceeding from the simplest case of the general unfree gauge algebra such that there is no off-shell disclosure, the Faddeev-Popov (FP) quantization recipe is deduced in the article [8]. Earlier, in the specific case of the unimodular gravity, the FP quantization recipe has been deduced in the paper $[9,10]$ making use of some nonlocal manipulations involving splitting the fields into longitudinal and transverse components and special gauge conditions. The set of ghosts involved in the general FP recipe in the case of unfree gauge parameters includes some extra variables comparing to the case of the unconstrained gauge symmetry. In the examples where the models with unfree gauge symmetry admit equivalent reformulations with unconstrained gauge parameters, the modified FP recipe can be explicitly reduced to the standard one [8].

In this paper, we propose the extension of the BV formalism to a general Lagrangian theory with unfree gauge symmetry. Our focus is at the algebraic aspects of the extension, while the subtleties are left aside concerning the functional aspects. In the next section, we describe the algebra of unfree gauge symmetry. Proceeding from the relaxed regularity assumptions such that admit irreducible unfree gauge symmetry, we deduce the basic structure relations of the gauge algebra including the most general off-shell terms. In Sect. 3, we propose the BV embedding for the Lagrangian theory with unfree gauge symmetry. This requires to introduce the 
set of ghosts and anti-fields adjusted for the unfree gauge algebra. The ghost and anti-field set is different from the theory with unconstrained gauge parameters. Given the set of fields and anti-fields, and the relaxed regularity conditions, we see that the classical master equation reproduces the structure relations of unfree gauge symmetry algebra. In Sect. 4, we consider the non-minimal sector and gauge fixing. As one can notice from the examples, the specifics of the unfree gauge symmetry is that the independent gauge fixing conditions break the relativistic symmetry, while the relativistic gauges are inevitably redundant even though the unfree gauge symmetry is irreducible. We give the non-minimal sector ghosts both for the independent and redundant gauges. For the theory without off-shell disclosure, we deduce the FP recipe by explicitly fixing the anti-fields by the gauge conditions. This is done both with independent and redundant gauges. In Sect. 5, we prove the existence theorem for the unfree gauge algebra. We use the homological perturbation theory (HPT) method. The key ingredient of the method is the Koszul-Tate differential. In the context of the existence theorem for the BV master equation, the Koszul-Tate differential has been first considered in the works $[4,11,12]$. The HPT method based on the Koszul-Tate complex was formulated in the article [13] as a tool for BV embedding of general Lagrangian gauge theories. This HPT procedure follows the pattern earlier suggested in the work [14] for the $\mathrm{BFV}^{2}$-BRST embedding of the Hamiltonian systems with reducible first class constraints. For the basics of the HPT applications to the BV formalism, we refer to the book [5]. In the case of the unfree gauge symmetry, the Koszul-Tate resolution of the mass shell differs from that for the gauge theory with unconstrained gauge parameters. Once the resolution is identified, the HPT allows one to construct the BV master action. In Sect. 6, we review the specific models with unfree gauge symmetry. After that, we consider one simple model to exemplify all the stages of the BV construction for the theory with unfree gauge symmetry. Section 7 includes concluding remarks.

Condensed notation In this article except for Sect. 6, where the specific field theory models are discussed, we adopt the DeWitt condensed notation. In this notation, the space of all the field histories is mimicked by the finite dimensional manifold $\mathcal{M}$, while the fields $\phi^{i}$ are treated as the local coordinates on $\mathcal{M}$. The index $i$ is "condensed", i.e. it comprises the space-time argument $x$ of the field, and all the discrete labels (like tensor, spinor, or flavor indices). The fields are supposed to obey certain boundary conditions when the spacial part of $x$ tends to infinity, and the asymptotics are understood as a part of definition of $\mathcal{M}$. In this article we imply zero boundary conditions. All the other variables, like gauge

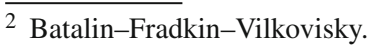

transformation parameters, ghosts, anti-fields, are treated like if they were the coordinates on the fibers of appropriate bundles over $\mathcal{M}$. Summation over the condensed index includes integration over the space-time argument. The matrices with condensed indices represent differential operators. For example, $\delta_{i j}$ includes $\delta(x-y)$ and delta symbol of discrete labels, so the matrix $M_{i j}$ can represent D'Alembertian with appropriate identification: $i=x, j=y, M_{i j}=\square \delta(x-y)$. In this notation, the Klein-Gordon equation reads $\left(M_{i j}+\right.$ $\left.m^{2} \delta_{i j}\right) \phi^{i}=0$. The local functionals, being integrals of the functions of the fields and their space-time derivatives $F(\phi)=\int d x \mathcal{F}\left(\phi, \partial_{x} \phi, \partial_{x}^{2} \phi, \ldots, \partial_{x}^{k} \phi\right)$ are mimicked by functions on $\mathcal{M}$, so the linear space of local functionals is treated like it was just a special subspace of smooth functions on $\mathcal{M}: F \in \mathcal{C}^{\infty}(\mathcal{M})$. We name the smooth functions of the fields and their derivatives $O\left(\phi, \partial_{x} \phi, \partial_{x}^{2} \phi, \ldots, \partial_{x}^{k} \phi\right)$ as local functions. We denote the algebra of local functions as $R(\mathcal{M})$. Any local function can be viewed as a local functional, so $R(\mathcal{M}) \subset \mathcal{C}^{\infty}(\mathcal{M})$. Derivatives with respect to $\phi^{i}$ are understood as functional derivatives of any local functional, including any local function. The condensed notation can be always unambiguously uncondensed. For further details of the condensed notation, we refer to the books $[5,15]$.

\section{Algebra of unfree gauge symmetry}

In this section, we at first address the issue of the Noether identities and their consequences, in the theories where the on shell vanishing local functions are not exhausted by the linear combinations of the left hand sides of the Lagrangian equations. In the second instance, we demonstrate that the modification of the Noether identities lead to the unfree gauge symmetry. Proceeding from the modified identities and unfree gauge symmetry, we deduce the higher structure relations of the unfree gauge symmetry algebra.

Consider the theory of fields $\phi^{i}$, with the action $S(\phi)$ being the local functional. The stationary surface of the action is defined by the Lagrangian equations

$\partial_{i} S(\phi)=0$.

The set of solutions of (1) is usually named the mass shell. We denote the mass shell $\Sigma$,

$\Sigma \subset \mathcal{M}, \quad \Sigma=\left\{\phi_{0} \in \mathcal{M} \mid \partial_{i} S\left(\phi_{0}\right)=0\right\}$

We use the sign $\approx$ to denote the on shell equality

$A(\phi) \approx B(\phi) \Leftrightarrow A\left(\phi_{0}\right)=B\left(\phi_{0}\right), \quad \forall \phi_{0} \in \Sigma, \quad A, B \in R(\mathcal{M})$. 
The local function $T(\phi)$ is considered as trivial if it vanishes on shell. In the classical field theory with equations of motion (1), every two local functions are considered equivalent if they differ by a trivial function,

$A \sim B \quad \Leftrightarrow \quad A-B \approx 0, \quad A, B \in R(\mathcal{M})$.

The trivial local functions constitute an ideal $I(\mathcal{M}) \subset R(\mathcal{M})$ in the algebra of local functions. The usual assumption of the general gauge field theory is that any trivial local function is spanned by the left hand sides of Lagrangian equations, i.e. the ideal $I$ is generated by $\partial_{i} S$,

$$
T(\phi) \approx 0 \quad \Leftrightarrow \quad T(\phi)=T^{i}(\phi) \partial_{i} S(\phi) .
$$

Once the condensed notation is used here, the coefficients $T^{i}(\phi)$ can describe the differential operators in space time argument, with the coefficients being local functions. This can be said in a slightly different wording. Any relation stating that the local function vanishes on shell should be the differential consequence of the Lagrangian equations.

The BV formalism [1-5] relays on the assumption (5) in several crucial aspects. Let us mention two of them. First, the restriction (5) is included into the set of sufficient conditions that ensure the existence of solution to the BV master equation. Second, even if the solution exists for the masterequation, while (5) does not hold true, the ideal $I$ of trivial local functions would not be isomorphic to the BRST-exact functions of zero ghost number. This would break the usual physical interpretation of the BRST cohomology groups.

The assumption (5) is violated in a number of field theories, see the examples in Sect. 6. Below, we elaborate on the general gauge symmetry algebra with a relaxed assumption (5).

If the left hand sides of the Lagrangian equations (1) cannot span every trivial local function, we assume that the ideal $I$ still admits a finite generating set. The ideal of the on-shell vanishing functions is supposed to be generated by $\partial_{i} S(\phi)$ and by a finite set of the other trivial functions $\tau_{a}(\phi)$,

$\tau_{a}(\phi) \approx 0, \quad \tau_{a}(\phi) \neq K_{a}^{i}(\phi) \partial_{i} S(\phi)$.

$T(\phi) \approx 0 \Leftrightarrow T(\phi)=V^{i}(\phi) \partial_{i} S(\phi)+V^{a}(\phi) \tau_{a}(\phi)$

Here, $V^{i}(\phi), V^{a}(\phi)$ stand for the differential operators in the space-time argument with the coefficients being local functions of the fields, as the condensed notations are applied. We name $\tau_{a}$, being the non-Lagrangian generating elements of the ideal of on-shell vanishing local functions, the completion functions of the Lagrangian system (1).

Note that the mass shell $\Sigma(2)$ is defined by the Lagrangian equations (1) while the relations $\tau_{a}(\phi) \approx 0$ do not restrict the solutions of (1), even though they are not differential consequences of the Eq. (1). The examples (see Sect. 6) demonstrate that this can happen in the field theories which do not reveal any inconsistency. Also notice that the completion functions $\tau_{a}(\phi)$ are defined modulo the left hand sides of the Lagrangian equations

$\tau_{a}^{\prime}(\phi) \sim \tau_{a}(\phi), \quad \tau_{a}^{\prime}(\phi)=\tau_{a}(\phi)+\theta_{a}^{i}(\phi) \partial_{i} S(\phi)$.

The generating elements of the ideal of on-shell vanishing local functions can be dependent, i.e. some of their linear combinations can vanish off shell:

$\Gamma_{\alpha}^{i}(\phi) \partial_{i} S(\phi)+\Gamma_{\alpha}^{a}(\phi) \tau_{a}(\phi) \equiv 0$

We consider these relations as the modified Noether identities.

The generators of the identities are considered equivalent $\Gamma \sim \Gamma^{\prime}$ if they differ by a trivial generator such that vanishes on shell:

$$
\begin{aligned}
\Gamma_{\alpha}^{\prime i}(\phi)-\Gamma_{\alpha}^{i}(\phi)= & E_{\alpha}^{i j}(\phi) \partial_{i} S(\phi) \\
& +E_{\alpha}^{i a}(\phi) \tau_{a}(\phi), \quad E_{\alpha}^{i j}=-E_{\alpha}^{j i} ; \\
\Gamma_{\alpha}^{\prime a}(\phi)-\Gamma_{\alpha}^{a}(\phi)= & E_{\alpha}^{a b}(\phi) \tau_{b}(\phi) \\
& -E_{\alpha}^{i a}(\phi) \partial_{i} S(\phi), \quad E_{\alpha}^{a b}=-E_{\alpha}^{b a} .
\end{aligned}
$$

Also notice that the different choice of completion functions (8) results in corresponding transformation of the modified Noether identity generators:

$$
\begin{aligned}
\tau_{a}(\phi) \mapsto \tau_{a}^{\prime}(\phi) & =\tau_{a}(\phi)+\theta_{a}^{i}(\phi) \partial_{i} S(\phi), \\
\Gamma_{\alpha}^{i} \mapsto \Gamma_{\alpha}^{\prime i} & =\Gamma_{\alpha}^{i}+\theta_{a}^{i}(\phi) \Gamma_{\alpha}^{a}
\end{aligned}
$$

We assume that the set of the modified Noether identities (9) is complete, i.e. any identity generator is spanned by the generators $\Gamma_{\alpha}$, modulo the equivalence relations (10), (11):

$$
\begin{aligned}
& L^{i}(\phi) \partial_{i} S(\phi)+L^{a}(\phi) \tau_{a}(\phi) \equiv 0 \Rightarrow L^{i}(\phi) \approx k^{\alpha}(\phi) \Gamma_{\alpha}^{i}(\phi), \\
& L^{a}(\phi) \approx k^{\alpha}(\phi) \Gamma_{\alpha}^{a}(\phi) .
\end{aligned}
$$

In this article, we further assume that the modified Noether identities (9) are not redundant

$K^{\alpha}(\phi) \Gamma_{\alpha}^{i}(\phi) \approx 0, K^{\alpha}(\phi) \Gamma_{\alpha}^{a}(\phi) \approx 0 \Leftrightarrow K^{\alpha}(\phi) \approx 0$,

i.e. there are no identities among the identities. This assumption can be relaxed. In the case of the theory with unfree gauge symmetry, the identities for identities could be accounted for, if they occurred, along the same lines as in the gauge theories with unconstrained gauge parameters and dependent gauge generators $[2,3,5]$. 
We adopt one more regularity assumption ${ }^{3}$ that all the completion functions (6) are essentially involved in the Noether identities (9), i.e.

$K_{a}(\phi) \Gamma_{\alpha}^{a}(\phi) \approx 0 \Leftrightarrow K_{a}(\phi) \approx 0$

Let us detail the off shell consequences of this condition. Once any on-shell vanishing local function is a linear combination of $\partial_{i} S$ and $\tau_{a}$, these relations mean that off shell $K_{a} \Gamma_{\alpha}^{a}$ reads

$K_{a} \Gamma_{\alpha}^{a} \equiv K_{\alpha}^{a} \tau_{a}+K_{\alpha}^{i} \partial_{i} S$

while $K_{a}$ is also spanned by the completion functions and the 1.h.s. of Lagrangian equations:

$K_{a}=L_{a}^{b} \tau_{b}+L_{a}^{i} \partial_{i} S$.

Substituting $K_{a}$ from (17) into (16) we get the Noether identity between the Lagrangian equations and completion functions (6). As any set of Noether identities is spanned by the generators $\Gamma$ (13), we arrive at the relations connecting the coefficients in the right hand sides of the relations (16) and (17):

$$
\begin{aligned}
K_{\alpha}^{a} & =L_{b}^{a} \Gamma_{\alpha}^{b}+M_{\alpha}^{\beta} \Gamma_{\beta}^{a}+A_{\alpha}^{a b} \tau_{b}+A_{\alpha}^{a i} \partial_{i} S, \\
A^{a b} & =-A^{b a} ; \\
K_{\alpha}^{i} & =L_{b}^{i} \Gamma_{\alpha}^{b}+M_{\alpha}^{\beta} \Gamma_{\beta}^{i}-A_{\alpha}^{a i} \tau_{a}+A_{\alpha}^{i j} \partial_{j} S, \\
A^{i j} & =-A^{j i} .
\end{aligned}
$$

Now, let us turn to the issue of gauge symmetry. Consider the infinitesimal transformation of $\mathcal{M}$

$\delta_{\epsilon} \phi^{i}=R_{\alpha}^{i}(\phi) \epsilon^{\alpha}$

The infinitesimal parameters $\epsilon^{\alpha}$ are labeled by the condensed index $\alpha$ that means they are functions of the space-time argument. The transformation (20) is understood as the gauge symmetry if it leaves the action invariant,

$\delta_{\epsilon} S(\phi) \equiv \epsilon^{\alpha} R_{\alpha}^{i} \partial_{i} S(\phi) \equiv 0$.

As the mass shell (2) is a stationary surface of the action, $\Sigma$ is automatically invariant under the gauge transformation. The gauge invariance condition (21) of the action means that certain linear combinations identically vanish of the Lagrangian equations. It is the second Noether theorem. Once the system does not obey the assumption (5), the most general Noether

\footnotetext{
3 This assumption is obeyed by all the presently known theories with unfree gauge symmetry. The examples are provided in Sect. 6 . It could be be relaxed, however this would lead to a more involved set of ghosts and anti-fields needed for the proper BV-BRST embedding of the theory. At the moment, this option seems having only the academic interest.
}

identity (9) involves both the Lagrangian equations (1) and completion functions (6). Any other Noether identity would be a linear combination of the ones from the generating set of identities (13). With this regard, one can identify the most general gauge symmetry generator $R_{\alpha}^{i}(20)$, (21) with the generator of Noether identity $\Gamma_{\alpha}^{i}(9)$. Then, under the transformation

$\delta_{\epsilon} \phi^{i}=\epsilon^{\alpha} \Gamma_{\alpha}^{i}(\phi)$

the action transforms as follows, given the identity (9):

$\delta_{\epsilon} S(\phi) \equiv \epsilon^{\alpha} \Gamma_{\alpha}^{i}(\phi) \partial_{i} S(\phi) \equiv-\epsilon^{\alpha} \Gamma_{\alpha}^{a}(\phi) \tau_{a}(\phi)$.

As all the operators $\Gamma_{\alpha}^{a}$ are assumed independent (14), this means the action is invariant provided for the gauge parameters are constrained by the equations

$\epsilon^{\alpha} \Gamma_{\alpha}^{a}(\phi)=0$

In the other wording, once the gauge variation (22) is unfree of the fields, with the gauge parameters obeying the Eq. (24), the action functional is invariant under the gauge transformation,

$\delta_{\epsilon} S(\phi) \equiv 0$

We see that the modified Noether identities (9) involving completion functions (6) result in the unfree gauge symmetry of theory.

In the gauge identities (9), the operators $\Gamma_{\alpha}^{i}$ and $\Gamma_{\alpha}^{a}$ are involved on an equal footing. However, they have different roles in the gauge symmetry transformations. The operator $\Gamma_{\alpha}^{i}$ generates the unfree gauge transformations (22), while $\Gamma_{\alpha}^{a}$ defines the equations (24) that restrict the gauge parameters. With this regard, we name $\Gamma_{\alpha}^{i}$ the generators of unfree gauge symmetry, while $\Gamma_{\alpha}^{a}$ are named the operators of gauge parameter constraints.

The local function(al) $O(\phi)$ is considered gauge invariant if the unfree gauge variation vanishes of $O(\phi)$ on shell,

$\delta_{\epsilon} O(\phi)=\epsilon^{\alpha} \Gamma_{\alpha}^{i}(\phi) \partial_{i} O(\phi) \approx 0$

Given the regularity conditions (7), (13), (14) imposed on the mass shell (2) and the gauge parameter constraints (24), this relation can be formulated off-shell, and without explicit involvement of the unfree parameters:

$$
\begin{aligned}
\delta_{\epsilon} O(\phi) \approx & 0 \Leftrightarrow \Gamma_{\alpha}^{i} \partial_{i} O(\phi)+V_{\alpha}^{i}(\phi) \partial_{i} S(\phi)+V_{\alpha}^{a}(\phi) \tau_{a}(\phi) \\
& +W_{a}(\phi) \Gamma_{\alpha}^{a}(\phi) \equiv 0 .
\end{aligned}
$$


Once the mass shell is invariant under the unfree gauge transformations, the ideal of the on-shell vanishing local functions is also gauge invariant,

$T(\phi) \approx 0 \Rightarrow \delta_{\epsilon} T(\phi) \approx 0$.

The relation (28) applies to any element of the ideal, including the completion functions, $\delta_{\epsilon} \tau_{a}(\phi) \approx 0$. Making use of (27) we get

$\Gamma_{\alpha}^{i}(\phi) \partial_{i} \tau_{a}(\phi)=R_{\alpha a}^{i}(\phi) \partial_{i} S(\phi)+R_{\alpha a}^{b}(\phi) \tau_{b}(\phi)+W_{a b}(\phi) \Gamma_{\alpha}^{b}(\phi)$.

The last term in this relation does not necessarily vanish on-shell. It has some specificity comparing to the unfree gauge transformation of the general on-shell vanishing function (27), (28): the structure coefficient $W_{a b}$ is on shell symmetric,

$W_{a b}(\phi)-W_{b a}(\phi) \approx 0$.

This property can be deduced as a differential consequence of the identities (9) with the account for the regularity conditions (15)-(17). Given the regularity conditions (15)-(19), the differential consequences of the identities (9) also define the commutators of gauge symmetry generators $\Gamma_{\alpha}^{i}$, and the action of the generators onto the operators of gauge parameter constraints $\Gamma_{\alpha}^{a}$ :

$$
\begin{aligned}
& \Gamma_{\alpha}^{i}(\phi) \partial_{i} \Gamma_{\beta}^{j}(\phi)-\Gamma_{\beta}^{i}(\phi) \partial_{i} \Gamma_{\alpha}^{j}(\phi)=U_{\alpha \beta}^{\gamma}(\phi) \Gamma_{\gamma}^{j}(\phi) \\
& \quad+E_{\alpha \beta}^{a j}(\phi) \tau_{a}(\phi)+E_{\alpha \beta}^{i j}(\phi) \partial_{i} S(\phi)+R_{\alpha a}^{j}(\phi) \Gamma_{\beta}^{a}(\phi) \\
& \quad-R_{\beta a}^{j}(\phi) \Gamma_{\alpha}^{a}(\phi) . \\
& \Gamma_{\alpha}^{i}(\phi) \partial_{i} \Gamma_{\beta}^{a}(\phi)-\Gamma_{\beta}^{i}(\phi) \partial_{i} \Gamma_{\alpha}^{a}(\phi)=U_{\alpha \beta}^{\gamma}(\phi) \Gamma_{\gamma}^{a}(\phi) \\
& \quad+R_{\alpha}^{a}(\phi) \Gamma_{\beta}^{b}(\phi)-R_{\beta}^{a}(\phi) \Gamma_{\alpha}^{b}(\phi)+E_{\alpha \beta}^{a b}(\phi) \tau_{b}(\phi) \\
& \quad-E_{\alpha \beta}^{a i}(\phi) \partial_{i} S(\phi),
\end{aligned}
$$

where the structure functions $E$ are antisymmetric, $E_{\alpha \beta}^{i j}=$ $-E_{\alpha \beta}^{j i}, E_{\alpha \beta}^{a b}=-E_{\alpha \beta}^{b a}$. The off-shell relations (31) mean, in particular, that any two unfree gauge transformations (22), (24) commute on-shell to another unfree gauge transformation. The off-shell relations (32) ensure that the equations (24) constraining the gauge parameters are on-shell gauge invariant themselves. This allows one to conclude that the unfree gauge symmetry transformations define on-shell integrable distribution. It foliates the mass shell into the gauge orbits, much like the gauge transformations would do if the gauge parameters were not constrained. This allows one to define physical observables in the usual way, as the equivalence classes (4) of the on-shell gauge invariant local function(al)s (26), (27). Any two observables are considered equivalent if they coincide on shell (4). Let us denote the subalgebra of on-shell gauge invariant local functions as $G(\mathcal{M})$, i.e.

$A \in G \quad \Leftrightarrow \quad \delta_{\epsilon} A \in I$,

Then the algebra of physical observables is understood as a quotient algebra $G / I$.

Let us summarize the most important specifics of the unfree gauge symmetry algebra. First, the generating set for the ideal of trivial local functions is not exhausted by the left hand sides of Lagrangian equations, it also includes the completion functions (6). The Noether identities are modified (9) also involving completion functions. Second, the gauge transformation parameters (22) are unfree, being constrained by the equations (24). Third, the regularity/completeness assumptions involve the equations of motion, completion functions, gauge generators and gauge parameter constraint operators (7), (13), (14). This specifics has to be accounted by an appropriate modification of the BV formalism such that can cover the systems with unfree gauge symmetry.

Remark Let us finalize the section with a remark on the possible alternative parametrization of the gauge symmetry such that have unconstrained gauge parameters. Notice the general solution to the equations constraining the gauge parameter (24) should involve the arbitrary functions. ${ }^{4}$ We denote these arbitrary functions $\omega^{A}$. The condensed index $A$ includes the space-time argument $x$, so $\omega^{A}$ are the arbitrary functions of $x$ indeed. They can be considered as the unconstrained gauge symmetry transformation parameters. In this setting, the solution to the equations (24) read

$\exists \Lambda_{A}^{\alpha}(\phi): \epsilon^{\alpha} \Gamma_{\alpha}^{a}(\phi) \approx 0 \Leftrightarrow \epsilon^{\alpha} \approx \Lambda_{A}^{\alpha}(\phi) \omega^{A}$,

The on-shell equality can be extended off shell,

$\Lambda_{A}^{\alpha} \Gamma_{\alpha}^{a}=E_{A}^{a b} \tau_{b}+E_{A}^{a i} \partial_{i} S, \quad E_{A}^{a b}=-E_{A}^{b a}$.

Introduce the new generators of gauge symmetry, being linear combinations of the original ones modulo on-shell vanishing terms:

$G_{A}^{i}=\Lambda_{A}^{\alpha} \Gamma_{\alpha}^{i}+E_{A}^{a i} \tau_{a}$.

Apply these generators to the action functional,

$G_{A}^{i} \partial_{i} S \equiv \Lambda_{A}^{\alpha} \Gamma_{\alpha}^{i} \partial_{i} S+E_{A}^{a i} \tau_{a} \partial_{i} S$.

\footnotetext{
4 The unfree gauge transformations should not be confused with socalled semi-local symmetries, see [16-18] and references therein. In both the cases, the transformation parameters have to obey the differential equations. The difference is that the solutions to the equations on the unfree parameters involve the arbitrary functions of $d$ coordinates in $d$ dimensional space, while in the semi-local case the arbitrary functions depend on $d-1$ coordinates or less.
} 
The identities (9) mean that $\Gamma_{\alpha}^{i} \partial_{i} S \equiv-\Gamma_{\alpha}^{a} \tau_{a}$. Substituting that to (37), and accounting for (35), we see

$G_{A}^{i} \partial_{i} S \equiv 0$

This means, the generators (36) define the unconstrained gauge symmetry of the action. Also, notice that the gauge symmetry transformations

$\delta_{\omega} \phi^{i}=G_{A}^{i} \omega^{a}$

can be reducible, the symmetry of symmetry can occur. In the article [19], it was shown that the unconstrained local parametrization of the gauge symmetry always exists for the linear field theories. This does not mean that any field theory model reduces to regular theory with (maybe reducible) unconstrained gauge symmetry. Even though the gauge symmetry transformations (39) involve unconstrained parameters, the completion functions do not disappear from the dynamics. They remain on shell vanishing, and thereby trivial, while they do not reduce to the linear combinations of the Lagrangian equations. So, if one tried to find the BV master action along the usual lines of BV formalism for the regular reducible gauge theory, and ignoring the completion functions, this can result in the contradictions. The matter is that the regularity conditions (5) are invalid if the the completion functions (6) admitted in the theory, while the existence theorem $[4,5]$ implies the relations (5) to hold true for the Lagrangian equations. So, the completion functions can obstruct the existence of the solution to the usual master equation. Furthermore, even if the solution exists, the completion functions will not correspond to the BRST-exact quantities, while they are trivial. This would violate the usual physical interpretation of the BRST cohomology. With this regard, in the next section we consider the construction of the BV formalism for the unfree gauge symmetry algebra with a proper account for the role of completion functions.

\section{Master equation}

Construction of the BV-BRST enbedding for the gauge system begins with the definition of the ghost and anti-field extension of the original set of the fields $\phi^{i}$. Below we provide some reasons for certain ghost/anti-field extension of $\mathcal{M}$ and formulate the master equation for the action. After that, we shall see that the master equation indeed reproduces the structure relations of the unfree gauge symmetry algebra deduced in the previous section. Then we shall see that the algebra of the gauge invariants of the original theory is mapped to the BRST cohohomology of the BV formalism. In the end of the section, we provide a reinterpretation of the constructed BV formalism in terms of "compensator fields".
In the next section we consider the gauge fixing in the BV formalism. A formal justification of the specific ghost and anti-field set is provided in Sect. 5, where we identify the Koszul-Tate differential for the mass shell of the theory with the unfree gauge symmetry and prove the existence theorem for the master equation.

To make the appropriate choice of ghosts and antifields for the theory with unfree gauge algebra, we proceed from the analogy with the BRST embedding of the not necessarily Lagrangian systems [6,7]. If the theory is defined just by the equations of motion (not necessarily Lagrangian), every equation is assigned with the anti-field whose ghost number is $-n-1$, where $n$ is the ghost number of the equation. Every generator of gauge identity is also assigned with the anti-field whose ghost number is $-n-2$, where $n$ is the ghost number of the equations involved in the identity. Every generator of the gauge symmetry is assigned with the ghost whose ghost number is $k+1$, where $k$ is the ghost number of the gauge parameter. Notice that for the non-Lagrangian systems, the gauge symmetries are not necessarily paired with the gauge identities, so the corresponding generators can be different, while in the Lagrangian case the same operator generates both gauge symmetry and gauge identity. In the Lagrangian systems with unfree gauge symmetry, the nonLagrangian pattern works well for introducing ghosts and anti-fields after two adjustments. The first is that the antifields are to be assigned to every element of the generating set for the ideal of on-shell vanishing local functions. This means, the anti-fields are introduced both for Lagrangian equations (1) and completion functions (6). We denote these anti-fields $\phi_{i}^{*}$ and $\xi_{a}^{*}$, respectively. The second is that the ghost $C^{\alpha}$ is assigned to every gauge symmetry generator $\Gamma_{\alpha}^{i}$ (22) even though the gauge parameters are unfree (24). The constraints on the gauge parameters are accounted for by imposing the same constraints on the ghosts:

$\Gamma_{\alpha}^{a}(\phi) C^{\alpha}=0$.

These equations are not involved in any gauge identity because of (15). We treat the equations for ghosts (40) on an equal footing with the other generating elements of $I$. This means, the anti-field has to be introduced for every ghost constraint (40). As the equations (40) are of the ghost number 1, the anti-fields should be assigned with the ghost number zero. We denote these anti-fields $\xi^{a}$. It does not mean the mere extension to the set of original fields $\phi^{i}$, because the $\mathrm{BV}$ formalism also implies one more grading: the resolution degree (also referred to as the anti-ghost number in some literature, e.g. in [5]). The anti-field $\xi^{a}$ has the resolution degree 1 , unlike $\phi^{i}$. And finally, the anti-fields $C_{\alpha}^{*}$ with ghost number -2 are assigned to the 
Table 1 Gradings of fields and anti-fields in a theory with unfree gauge symmetry

\begin{tabular}{llllrrr}
\hline Grading $\backslash$ variable & $\phi^{i}$ & $\xi^{a}$ & $C^{\alpha}$ & $\phi_{i}^{*}$ & $\xi_{a}^{*}$ & $C_{\alpha}^{*}$ \\
\hline$\varepsilon$ & 0 & 0 & 1 & 1 & 1 & 0 \\
gh & 0 & 0 & 1 & -1 & -1 & -2 \\
$\operatorname{deg}$ & 0 & $\mathbf{1}$ & 0 & 1 & 1 & 2 \\
\hline
\end{tabular}

modified Noether identities (9) alike the Lagrangian theory with unconstrained gauge parameters, even though the identities involve completion functions, unlike the usual case.

Let us introduce the notations for the gradings. The Grassmann parity is denoted $\varepsilon$, the ghost number is gh, and the resolution degree is deg. The assigned gradings are arranged in Table 1.

It is also convenient to use the collective notation

$\varphi^{I}=\left(\phi^{i}, \xi^{a}, C^{\alpha}\right), \quad \varphi_{I}^{*}=\left(\phi_{i}^{*}, \xi_{a}^{*}, C_{\alpha}^{*}\right)$

The ghost number grading of the variables is explained above. Now, we explain the reasons for assigning such resolution degrees to these variables. All the anti-fields paired to the generating elements of the ideal $I$ are assigned with the resolution degree 1 . It is the same principle as in the gauge theory with unconstrained gauge parameters, with one adjustment: the anti-fields are introduced not only for the original Lagrangian equations (1) $-\phi_{i}^{*}$, but also to the completion functions (6) $-\xi_{a}^{*}$, and to the equations constraining the ghosts $(40)-\xi^{a}$. The anti-fields are also introduced being paired with the Noether identities (9) $-C_{\alpha}^{*}$. These are assigned with the resolution degree 2 , much alike the theory with unconstrained gauge parameters.

Once the ghosts and anti-fields are introduced, this means we extended the manifold of original fields $\mathcal{M}$ to a $Z$-graded manifold $\overline{\mathcal{M}}$. The fields $\varphi, \varphi^{*}$ are considered as coordinates on $\overline{\mathcal{M}}$. The original equations of motion (1) and the constraints imposed on the ghosts (40) define the extended mass shell $\bar{\Sigma} \subset \overline{\mathcal{M}}$,

$$
\bar{\Sigma}=\left\{\left(\varphi_{0}, \varphi_{0}^{*}\right) \in \overline{\mathcal{M}} \mid \partial_{i} S\left(\phi_{0}\right)=0, C_{0}^{\alpha} \Gamma_{\alpha}^{a}\left(\phi_{0}\right)=0\right\}
$$

The algebra of local functions on $\overline{\mathcal{M}}$ is denoted as $\bar{R}$. It includes the ideal of the on-shell vanishing local functions, $\bar{I}$. The generating set for the ideal consists of the left hand sides of the Lagrangian equations (1), completion functions (6), and constraints on the ghosts (40),

$$
\begin{aligned}
A \in & \bar{I} \Leftrightarrow A=A^{i}\left(\varphi, \varphi^{*}\right) \partial_{i} S+A^{a}\left(\varphi, \varphi^{*}\right) \tau_{a} \\
& +A_{a}\left(\varphi, \varphi^{*}\right) C^{\alpha} \Gamma_{\alpha}^{a} .
\end{aligned}
$$

The classical theory is Lagrangian because the original mass shell is defined as a stationary surface of the action, even though the ideal $\bar{I}$ is not spanned by the left hand sides of Lagrangian equations. So, we are going to construct the BRST formalism in the Lagrangian BV form, representing the BRST differential as the anti-bracket with the master action $S\left(\varphi, \varphi^{*}\right)$.

Then, the first question is the definition of the antibracket for the local function(al)s $A\left(\varphi, \varphi^{*}\right)$. The set of fields (41) involves a pair of the anti-fields $\xi^{a}, \xi_{a}^{*}$ that does not have a counterpart in the theories without constraints on the gauge parameters, while all the other (anti-)fields have. As these variables are dual to each other, we find it natural to consider them as conjugate with respect to the anti-bracket. In the BV formalism, where the ghosts are unconstrained, once the variable has the positive resolution degree, being anti-field, the conjugate field always has zero degree. Both $\xi$ and $\xi^{*}$ are the anti-fields carrying positive degree, while they are conjugate to each other. As we shall see, this does not lead to any contradiction, though it may seem unusual. The other variables are naturally split into the conjugate pairs alike their counterparts in the BV formalism of the gauge theory without constraints onto parameters. All these reasons lead us to adopt the canonical anti-bracket with $\varphi^{I}$ being conjugate to $\varphi_{I}^{*}$ :

$$
\begin{aligned}
(A, B) & =\frac{\partial^{R} A}{\partial \varphi^{I}} \frac{\partial^{L} B}{\partial \varphi_{I}^{*}}-\frac{\partial^{R} A}{\partial \varphi_{I}^{*}} \frac{\partial^{L} B}{\partial \varphi^{I}}, \quad \varphi^{I}=\left(\phi^{i}, \xi^{a}, C^{\alpha}\right), \\
\varphi_{I}^{*} & =\left(\phi_{i}^{*}, \xi_{a}^{*}, C_{\alpha}^{*}\right) .
\end{aligned}
$$

The antibracket is Grassmann odd and it shifts the ghost number by one:

$\operatorname{gh}((A, B))=\operatorname{gh}(A)+\operatorname{gh}(B)+1, \quad \varepsilon((A, B))=\varepsilon(A)+\varepsilon(B)+1$.

The bracket is inhomogeneous with respect to the resolution degree.

The master action $S\left(\varphi, \varphi^{*}\right)$ is defined as an expansion with respect to the resolution degree

$S=\sum_{k=0} S_{k}, \quad \operatorname{gh}\left(S_{k}\right)=\varepsilon\left(S_{k}\right)=0, \quad \operatorname{deg}\left(S_{k}\right)=k$,

i.e. it is the graded expansion in the anti-fields $\xi^{a}, \xi_{a}^{*}, \phi_{i}^{*}, C_{\alpha}^{*}$. The initial term in this expansion is the original action

$S_{0}=S(\phi)$

Given the grading restrictions (46), the most general first and second resolution degree terms read

$S_{1}=\tau_{a} \xi^{a}+\left(\phi_{i}^{*} \Gamma_{\alpha}^{i}+\xi_{a}^{*} \Gamma_{\alpha}^{a}\right) C^{\alpha}$, 


$$
\begin{aligned}
S_{2}= & \frac{1}{2}\left(C_{\gamma}^{*} U_{\alpha \beta}^{\gamma}+\phi_{j}^{*} \phi_{i}^{*} E_{\alpha \beta}^{i j}+2 \xi_{a}^{*} \phi_{i}^{*} E_{\alpha \beta}^{i a}+\xi_{b}^{*} \xi_{a}^{*} E_{\alpha \beta}^{a b}\right) C^{\alpha} C^{\beta} \\
& -\xi^{b}\left(\phi_{i}^{*} R_{b \alpha}^{i}+\xi_{a}^{*} R_{b \alpha}^{a}\right) C^{\alpha}-\frac{1}{2} \xi^{b} \xi^{a} W_{a b},
\end{aligned}
$$

where all the expansion coefficients $\tau, \Gamma, U$, etc., can depend on the original fields $\phi$. The notations for the coefficients coincide with the corresponding structure functions in the relations of the unfree gauge symmetry algebra deduced in the previous section. It is not an abuse of notation. The structures with identical notation coincide indeed, as we shall see soon.

The master action (46) is defined by the BV master equation

$(S, S)=0$.

The solution to the master equation can be iteratively sought for by expanding the left hand side with respect to the resolution degree. The first two orders of the expansion of the equation involve only the first three orders of the master action, i.e. (47), (48), (49). Explicitly, this reads

$$
\begin{aligned}
(S, S)_{0}= & 2\left(\Gamma_{\alpha}^{a} \partial_{i} S+\Gamma_{\alpha}^{a} \tau_{a}\right) C^{\alpha}=0, \\
(S, S)_{1}= & 2 \xi^{a}\left(\Gamma_{\alpha}^{i} \partial_{i} \tau_{a}-R_{\alpha a}^{i} \partial_{i} S-R_{\alpha a}^{b} \tau_{b}-W_{a b} \Gamma_{\alpha}^{b}\right) C^{\alpha} \\
& -C^{\alpha} C^{\beta}\left(\phi _ { i } ^ { * } \left(\Gamma_{\alpha}^{j} \partial_{j} \Gamma_{\beta}^{i}-\Gamma_{\beta}^{j} \partial_{j} \Gamma_{\alpha}^{i}-U_{\alpha \beta}^{\gamma} \Gamma_{\gamma}^{i}-R_{\alpha a}^{i} \Gamma_{\beta}^{a}\right.\right. \\
& \left.+R_{\beta a}^{i} \Gamma_{\alpha}^{a}-E_{\alpha \beta}^{j i} \partial_{j} S-E_{\alpha \beta}^{i a} \tau_{a}\right) \\
& -\xi_{a}^{*}\left(\Gamma_{\alpha}^{j} \partial_{j} \Gamma_{\beta}^{a}-\Gamma_{\beta}^{j} \partial_{j} \Gamma_{\alpha}^{a}-U_{\alpha \beta}^{\gamma} \Gamma_{\gamma}^{a}-R_{\alpha b}^{a} \Gamma_{\beta}^{b}\right. \\
& \left.\left.+R_{\beta b}^{a} \Gamma_{\alpha}^{b}+E_{\alpha \beta}^{j a} \partial_{j} S-E_{\alpha \beta}^{a b} \tau_{b}\right)\right)=0 .
\end{aligned}
$$

The coefficients at all the independent monomials of the ghosts and anti-fields should be set to zero separately. In this way, we see that the zero order of the master equation expansion (51) is equivalent to the modified Noether identities (9) of unfree gauge symmetry algebra. The first order expansion of the master equation (52) is equivalent to the structure relations (29), (31), (32) of the unfree gauge symmetry algebra. One can see that the set of the ghosts and anti-fields of Table 1, and the boundary conditions (47), (48) for the master action, lead to the solution of the master equation (50) such that indeed corresponds to the theory with unfree gauge symmetry algebra. The solution to the master equation (50) exists in all the orders with respect to the resolution degree as we shall see in Sect. 5.

Once the master action is constructed, it defines the BRST differential

$s A=(A, S)$.

$s$ squares to zero because of the master equation (50) and Jacobi identity for the anti-bracket. It is Grassmann odd vector field of the ghost number 1 , $s^{2}=0, \quad \operatorname{gh}(s)=1, \quad \varepsilon(s)=1$.

The BRST differential can be expanded with respect to the resolution degree

$s=\delta+\gamma+\stackrel{(1)}{s} \ldots, \quad \operatorname{deg} \delta=-1, \quad \operatorname{deg} \gamma=0, \quad \operatorname{deg} \stackrel{(1)}{s}=1$.

As a consequence of (54), for the lower order terms of the expansion (55) we have

$s^{2}=0 \Rightarrow \delta^{2}=0, \quad \delta \gamma+\gamma \delta=0, \quad \gamma^{2}+(\delta \stackrel{(1)}{s}+\stackrel{(1)}{s} \delta)=0, \quad \ldots$

As one can see, $\delta$, being lowest resolution degree component of the BRST differential $s$, squares to zero, so it is a differential in itself. Explicitly, $\delta$ reads

$\delta A=-\frac{\partial^{R} A}{\partial \phi_{i}^{*}} \partial_{i} S-\frac{\partial^{R} A}{\partial \xi_{a}^{*}} \tau_{a}+\frac{\partial^{R} A}{\partial C_{\alpha}^{*}}\left(\phi_{i}^{*} \Gamma_{\alpha}^{i}+\xi_{a}^{*} \Gamma_{\alpha}^{a}\right)+\frac{\partial^{R} A}{\partial \xi^{a}} \Gamma_{\alpha}^{a} C^{\alpha}$.

It squares to zero, and this is equivalent to the modified Noether identities (9),

$\delta^{2} A=-\frac{\partial^{R} A}{\partial C_{\alpha}^{*}}\left(\Gamma_{\alpha}^{j} \partial_{j} S+\Gamma_{\alpha}^{a} \tau_{a}\right)=0$.

The differential $\delta$ can be understood as a Koszul-Tate resolution for the ideal $\bar{I}$ because of the two reasons. The first, any on-shell vanishing function of zero resolution degree is obviously $\delta$-exact

$$
\begin{aligned}
\operatorname{deg} A= & 0, A \in \bar{I} \Leftrightarrow A \equiv A^{i}(C, \phi) \partial_{i} S \\
& +A^{a}(C, \phi) \tau_{a}+A_{a}(\phi, C) \Gamma_{\alpha}^{a} C^{\alpha} \equiv \delta B,
\end{aligned}
$$

where $B=A^{a} \xi_{a}^{*}+A_{a} \xi^{a}+A^{i} \phi_{i}^{*}$. The second, $\delta$ is acyclic in the strictly positive resolution degree, i.e. the cohomology of $\delta$ is exhausted by zero resolution degree. This issue is addressed in Sect. 5. These two facts allow one to iteratively find all the higher orders of the master action by the HPT method.

Zero resolution degree contribution to the BRST differential (55) explicitly reads

$$
\begin{aligned}
\gamma A= & \frac{\partial^{R} A}{\partial \phi^{i}} C^{\alpha} \Gamma_{\alpha}^{i}+\frac{1}{2} \frac{\partial^{R} A}{\partial C^{\gamma}} U_{\alpha \beta}^{\gamma} C^{\alpha} C^{\beta} \\
& +\frac{\partial^{R} A}{\partial \xi^{a}}\left(\left(\phi_{i}^{*} E_{\alpha \beta}^{i a}-\xi_{b}^{*} E_{\alpha \beta}^{a b}\right) C^{\alpha} C^{\beta}-\xi_{b} R_{\alpha a}^{b} C^{\alpha}\right) \\
& -\frac{\partial^{R} A}{\partial \phi_{i}^{*}}\left(\xi^{a} \partial_{i} \tau_{a}+\left(\phi_{j}^{*} \partial_{i} \Gamma_{\alpha}^{j}+\xi_{a}^{*} \partial_{i} \Gamma_{\alpha}^{a}\right) C^{\alpha}\right) \\
& +\frac{\partial^{R} A}{\partial \xi_{a}^{*}}\left(\left(\phi_{i}^{*} R_{\alpha b}^{i}+\xi_{a}^{*} R_{\alpha b}^{a}\right) C^{\alpha}+\xi^{b} W_{a b}\right)
\end{aligned}
$$




$$
\begin{aligned}
& -\frac{\partial^{R} A}{\partial C_{\alpha}^{*}}\left(\left(U_{\alpha \beta}^{\gamma} C_{\gamma}^{*}+\phi_{i}^{*} \phi_{j}^{*} E_{\alpha \beta}^{i j}\right.\right. \\
& \left.\left.+2 \phi_{i}^{*} \xi_{a}^{*} E_{\alpha \beta}^{i a}+\xi_{a}^{*} \xi_{b}^{*} E_{\alpha \beta}^{a b}\right) C^{\beta}+\xi^{b}\left(\phi_{i}^{*} R_{\alpha b}^{i}+\xi_{a}^{*} R_{\alpha b}^{a}\right)\right) .
\end{aligned}
$$

It can be considered as a modification of the longitudinal differential of the usual gauge theory where the constraints (40) are not imposed on the ghosts. Because of (54), (55) $\gamma$ should anti-commute with $\delta$. Explicitly, this reads

$$
\begin{aligned}
(\delta \gamma+\gamma \delta) A= & -\frac{\partial^{R} A}{\partial \phi_{i}^{*}} \partial_{i}\left(\Gamma_{\alpha}^{i} \partial_{i} S+\Gamma_{\alpha}^{a} \tau_{a}\right) C^{\alpha} \\
& -\frac{\partial^{R} A}{\partial \xi_{a}^{*}}\left(\Gamma_{\alpha}^{i} \partial_{i} \tau_{a}-R_{\alpha a}^{i} \partial_{i} S-R_{\alpha a}^{b} \tau_{b}-W_{a b} \Gamma_{\alpha}^{b}\right) C^{\alpha} \\
& +\frac{1}{2} \frac{\partial^{R} A}{\partial \xi^{a}}\left(\Gamma_{\alpha}^{i} \partial_{i} \Gamma_{\beta}^{a}-\Gamma_{\beta}^{i} \partial_{i} \Gamma_{\alpha}^{a}-U_{\alpha \beta}^{\gamma} \Gamma_{\gamma}^{a}-R_{\alpha b}^{a} \Gamma_{\beta}^{b}\right. \\
& \left.+R_{\beta b}^{a} \Gamma_{\alpha}^{b}-E_{\alpha \beta}^{i a} \partial_{i} S-E_{\alpha \beta}^{a b} \tau_{b}\right) C^{\alpha} C^{\beta} \\
& -\frac{\partial^{R} A}{\partial C_{\alpha}^{*}}\left[\xi^{a}\left(\Gamma_{\alpha}^{i} \partial_{i} \tau_{a}-R_{\alpha a}^{i} \partial_{i} S-R_{\alpha a}^{b} \tau_{a}-W_{a b} \Gamma_{\alpha}^{b}\right) C^{\alpha}\right. \\
& +\phi_{i}^{*}\left(\Gamma_{\alpha}^{j} \partial_{j} \Gamma_{\beta}^{i}-\Gamma_{\beta}^{j} \partial_{j} \Gamma_{\alpha}^{i}-U_{\alpha \beta}^{\gamma} \Gamma_{\gamma}^{i}-R_{\alpha b}^{i} \Gamma_{\beta}^{b}+R_{\beta b}^{i} \Gamma_{\beta}^{b}\right. \\
& \left.-E_{\alpha \beta}^{i j} \partial_{j} S+E_{\alpha \beta}^{i a} \tau_{a}\right) \\
& +\xi_{a}^{*}\left(\Gamma_{\alpha}^{j} \partial_{j} \Gamma_{\beta}^{a}-\Gamma_{\beta}^{j} \partial_{j} \Gamma_{\alpha}^{a}-U_{\alpha \beta}^{\gamma} \Gamma_{\gamma}^{a}-R_{\alpha b}^{a} \Gamma_{\alpha}^{b}+R_{\beta b}^{a} \Gamma_{\alpha}^{b}\right. \\
& \left.\left.+E_{\alpha \beta}^{i a} \tau_{a}-E_{\alpha \beta}^{a b} \partial_{i} S\right)\right]=0,
\end{aligned}
$$

The anti-commutator vanishes because of the modified Noether identities (9), and structure relations of the unfree gauge symmetry algebra (29), (31), (32).

Once the BRST differential (53) has been constructed such that properly accounts for the completion functions (6) and constraints on the gauge parameters (24), one can expect that the physical observables of the theory are defined in the usual way, as the BRST cohomomology classes of the local function(al)s on $\overline{\mathcal{M}}$ of the ghost number zero

$$
\begin{aligned}
& H^{0}(s)=\operatorname{Ker}(s) / \operatorname{Im}(s)=\{A \in \bar{R}(\overline{\mathcal{M}}) \mid s A=0 ; \operatorname{gh} A=0 \\
& \left.\quad A \sim A^{\prime}, A-A^{\prime}=s B\right\} .
\end{aligned}
$$

In the original theory, the algebra physical observables is understood as the quotient of the subalgebra of on-shell gauge invariant local function(al)s (33) with respect to the ideal of the on-shell vanishing local function(al)s. This is alike the theories with unconstrained gauge parameters. The specifics of the case of unfree gauge symmetry is that the gauge invariance relations (27) involve the completion functions and gauge parameter constraint operators. These structures do not have any analogue in the theories with unconstrained gauge parameters. So, the question is to verify the isomorphism between the set of nontrivial gauge invariants of the original theory $G / I$ and BRST cohomology $H^{0}(s)$. Cohomological proof of the isomorphism is provided in Sect. 5. Here, we just demonstrate the explicit iterative construction of the BRST invariant corresponding to gaugeinvariant up to the first order with respect to the resolution degree. Consider the expansion

$\mathcal{O}\left(\varphi, \varphi^{*}\right)=\sum_{k=0} \stackrel{(k)}{\mathcal{O}}, \quad \operatorname{deg} \stackrel{(k)}{\mathcal{O}}=k, \quad \operatorname{gh} \stackrel{(k)}{\mathcal{O}}=0$,

where $\stackrel{(0)}{\mathcal{O}}=O(\phi)$ is the gauge invariant of the original theory (27). Given the expansions (55) and (63), the BRST invariance requirement connects the order $k+1$ with the lower orders of the invariant:

$$
s \mathcal{O}=0 \Leftrightarrow \delta \stackrel{(k+1)}{\mathcal{O}}+\gamma \stackrel{(k)}{\mathcal{O}}=-\sum_{m=1}^{k} \stackrel{(m)}{S} \stackrel{(k-m)}{\mathcal{O}}, \quad k=0,1,2, \ldots
$$

Consider the most general expression for $\mathcal{O}\left(\varphi, \varphi^{*}\right)$ up to the first order in the resolution degree

$$
\begin{aligned}
\mathcal{O}\left(\varphi, \varphi^{*}\right) & =O(\phi)+\stackrel{(1)}{\mathcal{O}}+\cdots \\
& =O(\phi)+C^{\alpha}\left(V_{\alpha}^{i} \phi_{i}^{*}+V_{\alpha}^{a} \xi_{a}^{*}\right)+W_{a} \xi^{a}+\cdots .
\end{aligned}
$$

Substitution of the ansatz (65) into the expansion (64) leads to the following expression in the leading order

$\delta \stackrel{(1)}{\mathcal{O}}+\gamma \stackrel{(0)}{\mathcal{O}}=C^{\alpha}\left(V_{\alpha}^{i} \partial_{i} S+V_{\alpha}^{a} \tau_{a}+W_{a} \Gamma_{\alpha}^{a}+\Gamma_{\alpha}^{i} \partial_{i} O\right)=0$.

This relation is obeyed by virtue of the gauge invariance condition for the local function $O(\phi)$ (27) upon identification of the expansion coefficients $V_{\alpha}^{i}, V_{\alpha}^{a}, W_{a}, \Gamma_{\alpha}^{a}, \Gamma_{\alpha}^{i}$ for $\stackrel{(1)}{\mathcal{O}}$ in the relation (65) with the corresponding structure functions in the relation (27).

So, any gauge invariant local function(al) $O(\phi)$ can be extended to the BRST invariant function(al) at least up to the first order with respect to the resolution degree. The extension has a natural ambiguity of the $\delta$-exact terms. This precisely corresponds to the equivalence relations (4) for the local function (4). So, one can see that the algebra of physical observables of original theory $G / I(33)$ is indeed isomorphic to the BRST cohomology $H^{0}(s)$ of zero ghost number to the first order in resolution degree. The higher orders exist, and they all can be iteratively constructed by the HPT method. This is explained in Sect. 5. Let us make one more remark concerning the solution to master equation. The variable $\xi^{a}$ has zero ghost number, and it is Grassmann even if the gauge parameters are even while it has the resolution degree one. This means, the expansion can be infinite in $\xi^{a}$. It might seem not a plausible iterative procedure for solving the master equation once it does not terminate in the final number 
of iterations. In fact, one can avoid explicitly finding all the orders in $\xi^{a}$. As we shall see in the next section, $\xi^{a}$ can be always fixed by an appropriate choice of gauge conditions, so only the first orders in $\xi^{a}$ can matter.

Remark Let us finalize the section by the remark on possible re-interpretation of the constructed BV-BRST formalism for the original theory with unfree gauge symmetry algebra. Let us consider the solution $S\left(\varphi, \varphi^{*}\right)$ of BV master equation (50) for the theory with unfree gauge symmetry algebra once it has been already constructed with the boundary conditions (47), (48). If we formally changed the resolution degree of $\xi^{a}$ from one to zero, this solution would remain a solution to the equation (50), because the definition of the anti-bracket (44) does not involve the resolution degree. Then, with respect to the modified resolution degree, the first two orders read of the master action

$S\left(\varphi, \varphi^{*}\right)=S^{\prime}(\phi, \xi)+C^{\alpha} R_{\alpha}^{i}(\phi, \xi) \phi_{i}^{*}+R_{\alpha}^{a}(\phi, \xi) \xi_{a}^{*}+\cdots$

This can be considered just as the master action for the theory of the fields $\phi, \xi$ with the action $S^{\prime}(\phi, \xi)$ and gauge symmetry generators $R_{\alpha}$. As a consequence of the master equation (50) obeyed by $S\left(\varphi, \varphi^{*}\right)$, the action $S^{\prime}(\phi, \xi)(67)$ is invariant under the gauge transformations

$$
\begin{aligned}
& \delta_{\epsilon} \phi^{i}=\epsilon^{\alpha} R_{\alpha}^{i}(\phi, \xi), \quad \delta_{\epsilon} \xi^{a}=\epsilon^{\alpha} R_{\alpha}^{a}(\phi, \xi), \\
& \delta_{\epsilon} S^{\prime} \equiv 0 \quad \forall \epsilon^{\alpha} .
\end{aligned}
$$

Notice that the gauge parameters of the transformations (68) are not constrained by any equations, it is the usual gauge symmetry. With this regard, the inclusion of the anti-fields $\xi^{a}$ to the equations constraining the ghosts (40) can be reinterpreted as inclusion of the compensator fields. By extending the set of original fields with the compensators, the original theory with the action $S(\phi)$, modified Noether identities (9), and the unfree gauge symmetry (22), (24) is converted into the theory of the original fields $\phi$ and compensators $\xi$ with the unconstrained gauge symmetry parameters (68). It is instructive to expand the action $S^{\prime}(\phi, \xi)$ and the gauge generators $R_{\alpha}(\phi, \xi)(68)$ in the power series with respect to the compensator fields $\xi^{a}$. The lower orders can be seen of the expansion from (47)-(49):

$$
\begin{aligned}
S^{\prime}(\phi, \xi) & =S(\phi)+\tau_{a}(\phi) \xi^{a}-\frac{1}{2} W_{a b}(\phi) \xi^{a} \xi^{b}+\ldots \\
R_{\alpha}^{i}(\phi, \xi) & =\Gamma_{\alpha}^{i}(\phi)+R_{\alpha a}^{i}(\phi) \xi^{a}+\ldots \\
R_{\alpha}^{a}(\phi, \xi) & =\Gamma_{\alpha}^{a}(\phi)+R_{\alpha b}^{a} \xi^{b}+\cdots
\end{aligned}
$$

All the expansion coefficients for the action and the gauge generators are defined by the unfree gauge symmetry algebra structures described in Sect. 2. In particular, $\tau_{a}$ are the completion functions (6); $W_{a b}$ are the structure coefficients in the relation (29); $\Gamma_{\alpha}^{i}, \Gamma_{\alpha}^{a}$ are defined by the modified Noether identities transformations (9) and play the roles of generators of unfree gauge symmetry transformations (22) and gauge parameter constraint operators (24); the coefficients $R_{\alpha a}^{i}, R_{\alpha b}^{a}$ are the structure functions in the commutation relations of the unfree gauge algebra (31), (32). So, by introduction of the compensator fields $\xi^{a}$, the completion functions and the related structure functions are absorbed by the action $S^{\prime}$, while the gauge parameter constraint operators and related structures are absorbed by the gauge generators of the extended theory. All the higher orders of the expansion (70), (71) exist, because the master equation has a solution.

The conversion of the theory with the unfree gauge symmetry parameters into an equivalent theory with extended set of fields, and without constraints on the gauge parameters is analogous to some extent to the conversion of the second class constraints into the first class ones [20,21], or to inclusion of the Stückelberg fields. The difference is that the second class constraints are absorbed by the first class ones, while in the theories with unfree gauge parameters, the operators of gauge parameter constraint operators are absorbed by the gauge generators, and the completion functions are absorbed by the action. One more common feature is that the HPT in both cases involves the conjugate variables such that have non-zero resolution degree, though in the conversion of Hamiltonian constraints the bracket is even, while in the unfree gauge theory it is odd.

From the viewpoint of the re-interpretation of the antifields $\xi^{a}$ as compensators, it is clear that the gauge conditions can be imposed such that fix $\xi^{a}$, and even force them to vanish. With these gauge imposed, it becomes unnecessary to explicitly find all the orders in $\xi$. It becomes sufficient to explicitly know the first order in $\xi$, once the higher orders can be excluded from the gauge fixed BRST invariant action by an appropriate gauge condition.

\section{Gauge fixing}

In this section, we briefly consider the gauge fixing procedure in the BV formalism of the theories with unfree gauge symmetry algebra.

The field-anti-field manifold $\overline{\mathcal{M}}$ (see the Table 1) has a structure of the odd cotangent bundle, and it is equipped with the canonical anti-bracket (44). From this viewpoint, it is the usual setup of the BV formalism. With this regards, the gauge fixing should mean the choice of the Lagrangian surface in $\overline{\mathcal{M}}$. It is defined by the equations

$\varphi_{I}^{*}=\frac{\partial \Psi}{\partial \varphi^{I}}, \quad \operatorname{gh}(\Psi)=-1, \quad \varepsilon(\Psi)=1$. 
The gauge fermion $\Psi$ involves the gauge fixing conditions imposed on the original fields and ghosts of so called nonminimal sector which are cohomologically trivial. The gauge invariance of the path integral is guaranteed by its independence from the choice of $\Psi$.

Let us first discuss the options of imposing the gauge fixing conditions on the original fields, and then turn to introducing the ghosts of non-minimal sector.

In principle, one can choose the gauge fixing conditions proceeding from the interpretation of the $\mathrm{BV}$ formalism for the unfree gauge theory as the BV theory of the equivalent gauge field theory of the original fields and the compensators and with unconstrained gauge parameters. Then, the independent gauge fixing conditions $\chi^{\alpha}(\phi, \xi)$ are imposed on the original fields and the compensators

$$
\begin{aligned}
& \chi^{\alpha}(\eta)=0, \quad A=(i, a), \quad \eta^{A}=\left(\phi^{i} ; \xi^{a}\right), \\
& \left.\operatorname{det}\left(\Gamma_{\alpha}^{A} \frac{\partial \chi^{\beta}}{\partial \eta^{A}}\right)\right|_{\xi=0} \neq 0 .
\end{aligned}
$$

The expression $\operatorname{det}\left(\Gamma_{\alpha}^{A} \frac{\partial \chi^{\beta}}{\partial \eta^{A}}\right)$ is the FP determinant of the gauge transformations (68), (71) evaluated at the point where the compensator fields vanish. Notice that the gauge transformations (68), (71) essentially affect the compensator fields $\xi^{a}$ in the sense that the gauge orbits are inevitably transverse to the level surface of the compensators $\xi^{a}$. This is seen from the explicit form of the transformation (68), (71) and irreducibility conditions for the operators of the gauge parameter constraints (15). This means, the gauge conditions (73) implicitly fix $\xi^{a}$, i.e. $\chi^{\alpha}(\phi, \xi) \approx 0 \Rightarrow \xi^{a} \approx f^{a}(\phi)$. This is much alike the gauge fixing in the theory with the gauge invariance such that includes the Stückelberg symmetry: the gauge conditions should fix the Stückelberg fields, at least implicitly. To make the immediate contact with the original theory without compensator fields, it would be instructive to impose the gauge conditions explicitly forcing $\xi^{a}$ to vanish. This can be done in two different ways, at least.

The first option is to choose the gauge conditions (73) explicitly separated into two subsets $\chi^{\alpha}=\left(\chi^{A}(\phi), \xi^{a}\right)$ :

$\alpha=(A, a) \quad \chi^{A}(\phi)=0, \quad \xi^{a}=0$,

where $\chi^{A}(\phi)$ are the independent gauge conditions imposed on the original fields. The latter can be understood as the gauge conditions of the original theory with unfree gauge symmetry parameters. Notice that the number of the independent gauge conditions $\chi^{A}(\phi)$ is less that the number of the generators of unfree gauge symmetry transformations by the number of constraints on the gauge parameters (24). Because of the numbers, the independent gauge conditions might be impossible to choose in explicitly relativistic covariant way, even if the gauge parameters are covariant. For example, if the gauge parameter is a vector subjected to the scalar con- straint, the number of independent conditions should be $d-1$. There are no tensorial structures of this dimension, while it seems unlikely to find $d-1$ independent scalars such that could serve as the gauge conditions. That is why it might be useful to consider redundant gauge fixing conditions if the explicit covariance is the issue upon gauge fixing - it is the second option.

Given the independent gauge conditions (74), corresponding Lagrange multipliers, ghosts, and anti-fields read:

$$
\begin{aligned}
\operatorname{gh} \pi_{A} & =\operatorname{gh} \pi_{a}=0, \quad \operatorname{gh} \bar{C}_{A}=\operatorname{gh} \bar{C}_{a}=-1, \\
\operatorname{gh} \bar{C}^{* A} & =\operatorname{gh} \bar{C}^{* a}=0 .
\end{aligned}
$$

The non-minimal action is constructed in the usual way,

$S_{\text {non-min }}=S+\bar{C}^{* A} \pi_{A}+\bar{C}^{* a} \pi_{a}$.

The gauge Fermion reads

$\Psi=\bar{C}_{A} \chi^{A}(\phi)+\bar{C}_{a} \xi^{a}$.

This allows one to fix the anti-fields $\varphi^{*}$ reducing the master action to the Lagrangian surface (72):

$S_{\Psi}=\left.S_{n o n-m i n}\right|_{\varphi * \mapsto \frac{\partial \Psi}{\partial \varphi}}$.

Given the gauge conditions $\xi^{a}=0$, the equations of the Lagrangian surface (72) include the relations $\xi_{a}^{*}=\bar{C}_{a}$, so the anti-fields $\xi_{a}^{*}$ a replaced in the gauge-fixed action $S_{\Psi}$ by the ghosts $\bar{C}_{a}$. Once the gauge conditions (74) kill off $\xi^{a}$, they can be put to zero in the gauge fixed action. After these reductions, the gauge fixed action reads

$$
\begin{aligned}
S_{\chi}\left(\phi^{i}, C^{\alpha}, \bar{C}_{A}, \pi^{A}\right)= & \left.S_{\Psi}\right|_{\xi} ^{a}=0=S(\phi)+\pi_{A} \chi^{A}(\phi) \\
& +\bar{C}_{A} \Gamma_{\alpha}^{i} \frac{\partial \chi^{A}}{\partial \phi^{i}} C^{\alpha}+\bar{C}_{a} \Gamma_{\alpha}^{a} C^{\alpha}+\cdots
\end{aligned}
$$

It can be considered as the most general BRST invariant action of the theory with unfree gauge symmetry when the gauge is fixed by imposing the independent conditions $\chi^{A}$ on the original fields. The ghosts $C^{\alpha}$ are assigned to all the gauge parameters, even though they are unfree. The anti-ghosts $\bar{C}_{A}$ are assigned to the gauge fixing conditions, while the antighosts $\bar{C}_{a}$ are assigned to all the constraints on the gauge parameters (24). The first item in $S_{\chi}$ is the original action, the second one fixes the gauge. The third item is the FP term, while the fourth one can be considered as a product of the constraints on the ghosts (40) to the Lagrange multiplier $\bar{C}_{a}$. The higher order terms can involve, in principle, the squares and higher orders of $\bar{C}_{a}$, so it is not a true multiplier.

The number of the independent gauge conditions $\chi^{A}(\phi)$ (74) is less than the number of gauge parameters, so they most likely would break explicit relativistic symmetry. If the 
explicit Poincaré or AdS symmetry is needed, then the number of gauge conditions should coincide with the number of gauge parameters. Once the parameters are unfree, the gauge conditions should be redundant. In a slightly different wording, in the theories with unfree gauge invariance, the Coulomb-type gauge conditions can be independent, while the Lorentz-type ones cannot. With this regard, it is useful to consider the redundant gauge conditions imposed on the original fields, and fix the compensators,

$\chi^{\alpha}(\phi)=0, \quad \xi^{a}=0$,

The fact that the gauge conditions $\chi^{\alpha}(\phi)$ are redundant means

$\exists \Delta_{\alpha}^{a}(\phi): \Delta_{\alpha}^{a} \chi^{\alpha} \approx 0$

The null-vectors $\Delta$ are supposed irreducible,

$\exists \Lambda_{b}^{\alpha}: \quad \operatorname{det}\left(M_{b}^{a}\right) \neq 0, \quad M_{b}^{a}=\Lambda_{b}^{\alpha} \Delta_{\alpha}^{a}$.

The redundant gauge conditions are usually imposed in the theories with reducible gauge symmetries [2,3]. With the unfree the non-minimal sector anti-ghosts are introduced for the redundant gauges by the same scheme, though it is asymmetric with respect to the ghosts of the minimal sector, unlike the reducible gauge symmetry. Given the reducible gauge conditions, the non-minimal sector read

$$
\begin{aligned}
\operatorname{gh} \bar{C}_{a}= & \operatorname{gh} \bar{C}_{\alpha}=\operatorname{gh} \zeta_{a}^{*}=\operatorname{gh} \pi^{* \alpha}=\operatorname{gh} \pi^{* a}=-\operatorname{gh} \lambda^{a}=-1 ; \\
& \operatorname{gh} \lambda_{a}^{*}=-2 ; \\
\operatorname{gh} \zeta^{a}= & \operatorname{gh} \pi_{\alpha}=\operatorname{gh} \bar{C}^{* \alpha}=\operatorname{gh} \bar{C}^{* a}=0 .
\end{aligned}
$$

Here, $\bar{C}_{\alpha}$ are the anti-ghosts to the redundant gauge conditions $\chi^{\alpha}(\phi)$, anti-ghosts $\bar{C}_{a}$ correspond to the conditions fixing $\xi^{a}, \pi_{\alpha}$ are the Lagrange multipliers to the redundant gauge conditions, $\lambda^{a}$ are the ghosts for the gauge symmetry of the Lagrange multipliers $\pi_{\alpha}$, and $\zeta^{a}$ are the Lagrange multipliers to the gauge conditions imposed on $\pi_{\alpha}$. All the non-minimal sector variables are introduced with their antifields. In the master action these variables are included in the same way as in the theories with reducible gauge symmetries,

$S_{n o n-\min }=S\left(\varphi, \varphi^{*}\right)+\bar{C}^{* \alpha} \pi_{\alpha}+\bar{C}^{* a} \pi_{a}+\lambda^{a} \zeta_{a}^{*}$

The gauge Fermion reads

$\Psi=\bar{C}_{\alpha}\left(\chi^{\alpha}+\Lambda_{b}^{\alpha} \zeta^{b}\right)+\bar{C}_{a} \xi^{a}$.

On the Lagrange surface (72) defined by $\Psi$ all the antifields are excluded from the master action. In particular, the antifields $\xi_{a}^{*}$ are replaced by $\bar{C}_{a}$. The conjugate fields $\xi^{a}$ are killed off by the gauge conditions (80). As a result, the gauge fixed action depends only on the original fields, ghosts to the unfree gauge symmetry, and extended set of the anti-ghosts:

$$
\begin{aligned}
S_{\chi}= & \left.S_{n o n-\min }\right|_{\varphi^{*}=\frac{\partial \Psi}{\partial \varphi} ; \xi^{a}=0}=S(\phi)+\pi_{\alpha} \chi^{\alpha}(\phi)+\zeta^{b} \Lambda_{b}^{\alpha} \pi_{\alpha} \\
& +\bar{C}_{b} \Gamma_{\alpha}^{b} C^{\alpha}+\bar{C}_{\beta} \Gamma_{\alpha}^{i} \frac{\partial \chi^{\beta}}{\partial \phi^{i}} C^{\alpha}+\lambda^{b} \Lambda_{b}^{\alpha} \bar{C}_{\alpha}+\cdots
\end{aligned}
$$

The gauge fixed action begins with the original one. Then, it involves two gauge fixing terms. The first one involves the redundant gauge conditions $\chi^{\alpha}$, while the second item fixes the gauge for the multipliers $\pi_{\alpha}$. The second line in (87) includes the modification of the FP action term for the theory with unfree gauge symmetry and redundant gauge conditions.. If the gauge conditions $\chi^{\alpha}$ were explicitly split into the independent ones, and the identical zeros $\chi^{\alpha}=\left(\chi^{A} ; \chi^{a} \equiv 0\right)$, the extra ghosts $\lambda^{a}$, the corresponding multipliers $\pi_{a}$, and the variables $\zeta^{a}$ would decouple from the other terms, and their contributions would cancel each other in the path integral. The remaining action would coincide with the expression (79) corresponding to the independent gauge conditions.

\section{The unique existence of solution to the master equation}

In Sect. 3, the master equation has been formulated for the theories with unfree gauge symmetry algebra. We have explicitly found the solution in the second order approximation with respect to the resolution degree (48), (49). Now, we consider the existence problem in any order. At first, we shall see that the problem can be brought to the usual HPT setup with respect to the Koszul-Tate differential (57). Then, we consider the cohomology of $\delta$. The differential $\delta$ can be understood a resolution for the ideal $\bar{I}$ (43) of the on-shell vanishing local function(al)s. The ideal $\bar{I}$ is generated not only by the left hand sides of the Lagrangian equations, but also by the completion functions (6), (7), and by the constraints imposed on the ghosts (40). Once the generating set is different for $\bar{I}$ comparing to the corresponding ideal in the case of the Lagrangian theory with unconstrained gauge symmetry parameters, the cohomology of the differential has to be examined in this case. We shall demonstrate that $\delta$ is acyclic in the strictly positive resolution degrees. Given the acyclicity of $\delta$, and relation (56), we shall see that the master equation can be iteratively solved by the usual HPT tools. And finally, we shall address the issue of physical observables.

The existence problem reads: given the first two orders

$$
\begin{aligned}
S_{0}\left(\varphi, \varphi^{*}\right)= & S(\phi), \quad S_{1}\left(\varphi, \varphi^{*}\right)=\tau_{a}(\phi) \xi^{a} \\
& +\left(\phi_{i}^{*} \Gamma_{\alpha}^{i}(\phi)+\xi_{a}^{*} \Gamma_{\alpha}^{a}(\phi)\right) C^{\alpha},
\end{aligned}
$$


in the expansion of the master action with respect to the resolution degree

$S\left(\varphi, \varphi^{*}\right)=\sum_{k=0} S_{k}, \quad \operatorname{gh}\left(S_{k}\right)=\varepsilon\left(S_{k}\right)=0, \quad \operatorname{deg}\left(S_{k}\right)=k$,

to iteratively solve the master equation (50) for all the higher orders $S_{k}, k>1$.

The solution goes along the usual lines of the BV method. We substitute the expansion (89) into the master equation $(S, S)=0$, and expand the left hand side with respect to the resolution degree. In zero order, the equation is obeyed because of the modified Noether identities (9), as is noticed in Sect. 2, see (51). In the the order $k \geq 1$, it has the structure

$\delta S_{k+1}=B_{k}, \quad \operatorname{gh} B_{k}=1 \quad \operatorname{deg} B_{k}=k$,

where $B_{k}$ is constructed of the derivatives of $S_{l}, l \leq k$. The solution will exist for $S_{k+1}$ if $B_{k}$ is $\delta$-exact. Because of the Jacobi identity for the anti-bracket, one can see that $B_{k}$ is $\delta$-closed:

$\delta B_{k}=0$.

So, the existence problem reduces to the issue of $\delta$ cohomology in the positive resolution degrees.

Let us demonstrate that the Koszul-Tate differential is acyclic in the class of functions with deg $>0$. For simplicity, we consider the functions of the ghost number one, and resolution degree one. The higher degrees are treated in a similar way, while the manipulations are more lengthy. The most general $B_{1}^{1}$ reads

$$
\begin{aligned}
& \operatorname{gh}\left(B_{1}^{1}\right)=1, \quad \operatorname{deg}\left(B_{1}^{1}\right)=1 \Leftrightarrow B_{1}^{1}=C^{\alpha} B_{a \alpha} \xi^{a} \\
& +\frac{1}{2} C^{\alpha} C^{\beta}\left(B_{\alpha \beta}^{i} \phi_{i}^{*}+B_{\alpha \beta}^{a} \xi_{a}^{*}\right) .
\end{aligned}
$$

Suppose $B_{1}^{1}$ is $\delta$-closed. Then, the structure functions $B_{a \alpha}(\phi), B_{\alpha \beta}^{a}, B_{\alpha \beta}^{i}(\phi)$ obey the relations:

$\delta B_{1}^{1}=C^{\alpha} C^{\beta}\left(\Gamma_{\alpha}^{a} B_{a \beta}+B_{\alpha \beta}^{i} \partial_{i} S+B_{\alpha \beta}^{a} \tau_{a}\right)=0$.

These relations involve the left hand sides of the Lagrangian equations, the completion functions (6), and the operators of gauge parameter constraints (24). These quantities are involved in the modified Noether identities (9), and they are assumed to obey the regularity and completeness conditions, see (7), (13), (14). These relations have the consequences (15)-(18). Given the regularity and completeness assumptions for the unfree gauge symmetry algebra, and their consequences, the relation (93) means

$$
\begin{aligned}
& \exists A_{(a b)}, A_{b \alpha}^{i}, A_{b \alpha}^{a}, U_{\alpha \beta}^{\gamma}, E_{\alpha \beta}^{[i j]}, E_{\alpha \beta}^{i a}, E_{\alpha \beta}^{[a b]}: \\
& B_{a \alpha}=A_{a b} \Gamma_{\alpha}^{b}+A_{a \alpha}^{i} \partial_{i} S+A_{a \alpha}^{b} \tau_{b} ; \\
& B_{\alpha \beta}^{i}=\Gamma_{[\alpha}^{a} A_{\beta] a}^{i}+U_{\alpha \beta}^{\gamma} \Gamma_{\gamma}^{i}+E_{\alpha \beta}^{[i j]} \partial_{j} S+E_{\alpha \beta}^{[i a]} \tau_{a} ; \\
& B_{\alpha \beta}^{a}=\Gamma_{[\alpha}^{b} A_{\beta] b}^{a}+U_{\alpha \beta}^{\gamma} \Gamma_{\gamma}^{a}-E_{\alpha \beta}^{a j} \partial_{j} S+E_{\alpha \beta}^{[a b]} \tau_{b} .
\end{aligned}
$$

Once the structure coefficients $B_{a \alpha}, B_{\alpha \beta}^{i}, B_{\alpha \beta}^{a}$ read as above, $B_{1}^{1}$ is $\delta$-exact:

$B_{1}^{1}=\delta D_{2}^{0}, \quad \operatorname{gh} D_{2}^{0}=0, \quad \varepsilon D_{2}^{0}=0, \quad \operatorname{deg} D_{2}^{0}=2$,

where

$$
\begin{aligned}
D_{2}^{0}= & \frac{1}{2} A_{a b} \xi^{a} \xi^{b}+C^{\alpha} \xi^{b}\left(\phi_{i}^{*} A_{b \alpha}^{i}+A_{b \alpha}^{a} \xi_{a}^{*}\right) \\
& +\frac{1}{2} C^{\alpha} C^{\beta}\left(U_{\alpha \beta}^{\gamma} C_{\gamma}^{*}+E_{\alpha \beta}^{i j} \phi_{i}^{*} \phi_{j}^{*}+E_{\alpha \beta}^{i a} \phi_{i}^{*} \xi_{a}^{*}\right. \\
& \left.+E_{\alpha \beta}^{a b} \xi_{a}^{*} \xi_{b}^{*}\right) .
\end{aligned}
$$

This means, there exists $S_{2}=D_{2}^{0}+\delta \Psi, \operatorname{gh} \Psi=-1, \operatorname{deg} \Psi=$ 3 . Once $\delta$ is acyclic in the higher resolution degrees, this proves the unique ${ }^{5}$ existence of all the higher order items in the expansion (89).

Let now discuss the details of construction of the BRST invariant physical observables by means of the HPT method. The problem reads as follows: given the first two orders

$\mathcal{O}_{0}=O(\phi), \quad \mathcal{O}_{1}=C^{\alpha}\left(V_{\alpha}^{i} \phi_{i}^{*}+V_{\alpha}^{a} \xi_{a}^{*}\right)+W_{a} \xi^{a}$,

in the expansion of the physical observable in the resolution degree (63), to iteratively solve equation (64) for all the higher orders $\mathcal{O}_{k}, k>1$.

The solution goes along the usual lines of the BV method, given the acyclicity of $\delta$ in positive resolution degrees. For $k=1$ Eq. (64) is satisfied because of the condition (66). For $k>1$, the structure of Eq. (65) reads

$\delta \mathcal{O}_{k+1}=\mathcal{B}_{k}, \quad \operatorname{gh} \mathcal{B}_{k}=1, \quad \operatorname{deg} \mathcal{B}_{k}=k$,

where $\mathcal{B}_{k}$ is constructed of the derivatives of $\mathcal{O}_{l}, l \leq k$. The solution will exist for $\mathcal{O}_{k+1}$ if $\mathcal{B}_{k}$ is $\delta$-exact. Because of the Jacobi identity for the anti-bracket, one can see that $\mathcal{B}_{k}$ is $\delta$-closed:

$\delta \mathcal{B}_{k}=0$

The quantity $\mathcal{B}_{k}$ is $\delta$-exact because the Koszul-Tate differential $\delta$ is acyclic in any positive resolution degree. This proves the existence of the BRST invariant physical observables. As in the usual BV formalism, the BRST invariant is unique for

\footnotetext{
$\overline{5}$ Up to the $\delta$-exact terms. This is a natural ambiguity related to the fact, that the structure functions of the gauge algebra are defined modulo the on shell vanishing contributions.
} 
any original physical observable, modulo BRST-exact terms. So the factor algebra $R / I$ of the nontrivial gauge invariant function(al)s is isomorphic to the BRST cohomology group of zero ghost number.

\section{Examples}

The best known example of unfree gauge symmetry is provided by the volume preserving diffeomorphism, $T$-diff. $T$ diff is the gauge symmetry of unimodular gravity and various modifications, see [22-31] and references therein. Once the unimodularity condition det $g_{\mu \nu}=-1$ is imposed, the gauge variation $\delta_{\epsilon} g_{\mu \nu}=\nabla_{\mu} \epsilon_{\nu}+\nabla_{\nu} \epsilon_{\mu}$ is unfree of the metric, as the transformation have to be consistent with the fixed volume. This means, the transformation parameter $\epsilon^{\mu}(x)$ is constrained by the transversality condition,

$\delta_{\epsilon} \operatorname{det} g_{\mu \nu}=0 \Leftrightarrow \partial_{\mu} \epsilon^{\mu}=0$.

A similar phenomenon is known in the model of the massless free spin 2 described by traceless tensor field [32,33]. In this case, the Fierz-Pauli gauge symmetry parameter has to obey the transversality condition (103). The extension is known of this class of the massless spin models to the fields of any spin $s$ [34]. The gauge parameters, being the symmetric traceless tensors of rank $s-1$ turn out unfree again. The Maxwelllike models $[35,36]$ of higher spins involve tracefull tensors, whose gauge symmetry is parameterized by the tracefull tensors of a lower rank, while the differential equations are still imposed on the gauge parameters. So, the phenomenon of the unfree gauge symmetry is not necessarily related to any constraint (like trace condition) imposed on the fields.

In all the above cases, the regularity assumption (5) is invalid, while the relaxed conditions (6), (7) hold true, at least modulo some subtleties related to global degrees of freedom. In the model of spin 2 [32,33], the field equations for the Minkowski space traceless tensor $h^{\mu \nu}$ has a differential consequence:

$\partial_{\lambda} \partial_{\mu} \partial_{\nu} h^{\mu \nu} \approx 0$

This means, $\partial_{\mu} \partial_{\nu} h^{\mu \nu} \approx$ const. The usual boundary conditions imply the fields to vanish on the spacial infinity in Minkowski space. ${ }^{6}$ With zero boundary conditions, the constant should vanish, so we have

\footnotetext{
6 The boundary conditions should not be confused with Cauchy data which define the initial values of the fields and their time derivatives on some space-like hyper-surface, not at spacial infinity. Zero boundary conditions do not contradict to nontrivial Cauchy data.
}

$\tau \equiv \partial_{\mu} \partial_{\nu} h^{\mu \nu} \approx 0$

The quantity $\tau$ is a function of derivatives of the fields, it is local. It vanishes on-shell, given the boundary conditions. However, the relation $\tau \approx 0$ is not a differential consequence of the Lagrangian equations. So, we have the on-shell trivial local quantity which does not reduce to a linear combination of the Lagrangian equations and their derivatives. It should be considered as a completion function as defined by relations (6). Notice that the higher spin extensions [34] of the spin two traceless tensor field theory [32,33] also reveal a existence of the completion functions corresponding to the definition (6). The Maxwell-like models of higher spins [35,36] also have the similar local quantities such that vanish on shell and do not reduce to the linear combinations of the Lagrangian equations. Detailed explanations of these quantities can be found in the reference [36]. In the unimodular gravity, the field equations have the differential consequence

$\partial_{\mu} R \approx 0 \Rightarrow R \approx \Lambda, \quad \Lambda=$ const,

where $R$ is the Ricci curvature of the unimodular metric. This means,

$R \approx \Lambda, \quad \Lambda=$ const

If the space is supposed to be asymptotically flat, $\Lambda$ has to be zero. If the metric has the (A)dS asymptotics, $\Lambda$ is a fixed constant being defined by the asymptotic (A)dS curvature radius. So, with any fixed asymptotics of the metric, the completion function (6) would be $\tau=R-\Lambda$. If the asymptotics is not fixed of the metrics, then the constant $\Lambda$ can be considered as a modular parameter involved in the same completion function $\tau=R-\Lambda$. The subtleties concerning the account for the modular parameters are beyond the scope of this paper. We can only notice, that once the completion function is explicitly involved in the formalism, the presence of the modular parameters is also made explicit that could facilitate the study of their impact on the dynamics.

Now, let us consider the specific model to exemplify the general formalism. The linearized unimodular gravity is theory of second-rank symmetric traceless tensor field $h_{\mu \nu}(x)$ with the action functional

$$
\begin{aligned}
S_{0} & =\int L d^{4} x \\
L & =\frac{1}{2}\left(\partial_{\mu} h_{v \rho} \partial^{\mu} h^{v \rho}-2 \partial^{\mu} h_{\mu \rho} \partial_{\nu} h^{v \rho}\right), \quad h_{\mu}^{\mu} \equiv 0 .
\end{aligned}
$$

The gauge symmetry of the action is the linearized volumepreserving diffeomorphism T-diff,

$\delta_{\epsilon} h^{\mu \nu}=\partial^{\mu} \epsilon^{\nu}+\partial^{v} \epsilon^{\mu}, \quad \partial_{\mu} \epsilon^{\mu}=0$, 
where $\epsilon^{\mu}$ is the transformation parameter. The gauge symmetry is constrained because the transformation parameter has to obey the transversality condition.

The Lagrangian equations read:

$$
\frac{\delta S}{\delta h^{\mu \nu}}=-\left(\square h_{\mu \nu}-\partial_{\mu} \partial^{\rho} h_{\rho \nu}-\partial_{\nu} \partial^{\rho} h_{\rho \mu}+\frac{1}{2} \partial_{\rho} \partial_{\sigma} h^{\rho \sigma}\right) \approx 0 .
$$

Taking the divergence of the equations, we arrive at the consequence $\partial_{\mu} \tau \approx 0$, where $\tau=\partial_{\mu} \partial_{\nu} h^{\mu \nu}$. Given the boundary conditions that the fields are vanishing at the space infinity, we arrive at (105). The completion function $\tau$ is not a linear combination of the right hand sides of the Lagrangian equations. One can see that the completion function is preserved by the $T$-diffs,

$\delta_{\epsilon} \tau=2 \square \partial_{\mu} \epsilon^{\mu}$.

The gauge invariance of the completion function holds true only with account of the constraint on gauge parameter, so the structure function $W$ (29), (30) does not vanish. In the case at hands

$W=2 \square$.

This structure function is symmetric (cf. (30)), because the D'Alembert operator is formally self-adjoint, as it should be.

There is the modified Noether identity (9) involving the Lagrangian equations (110) and completion function (105),

$\partial^{\nu} \frac{\delta S}{\delta h^{\mu \nu}}-\frac{1}{2} \partial^{\mu} \tau \equiv 0$.

The gauge identity generators have the form

$\Gamma_{\rho}^{\mu v}=\delta_{\rho}^{\mu} \partial^{v}+\delta_{\rho}^{\mu} \partial^{v}, \quad \Gamma_{\rho}=-\partial_{\rho}$.

As we see, these quantities determine the gauge transformation (109) of the theory (108) and constraint on parameter by the rule (22), (24). The gauge generators are field independent, so the structure functions $R_{\alpha a}^{i}, R_{\alpha a}^{b}, E_{\alpha \beta}^{i j}, E_{\alpha \beta}^{i a}, E_{\alpha \beta}^{a b}$ (31), (32) vanish identically.

To construct the BV-BRST embedding of the linearized unimodular gravity, we introduce the ghosts and anti-fields following the general prescription (cf. Table 1):

$h^{\mu \nu}(x), \quad h^{*}{ }_{\mu \nu}(x), \quad C^{\mu}(x), \quad C_{\mu}^{*}(x), \quad \xi(x), \quad \xi^{*}(x)$.

The condensed indices labeling the fields $\phi^{i}$, gauge parameters $\epsilon^{\alpha}$, and the completion functions $\tau_{a}$ are uncondensed in the following way: $i=\{\mu \nu, x\} ; \alpha=\{\mu, x\}, a=\{x\}$, where
Table 2 Gradings of fields and anti-fields in the linearized unimodular gravity

\begin{tabular}{llllrrr}
\hline Grading $\backslash$ variable & $h^{\mu \nu}$ & $\xi$ & $C^{\mu}$ & $h_{\mu \nu}^{*}$ & $\xi^{*}$ & $C_{\mu}^{*}$ \\
\hline$\varepsilon$ & 0 & 0 & 1 & 1 & 1 & 0 \\
gh & 0 & 0 & 1 & -1 & -1 & -2 \\
$\operatorname{deg}$ & 0 & 1 & 0 & 1 & 1 & 2 \\
\hline
\end{tabular}

$x$ is the space-time argument. The gradings of the fields and anti-fields are arranged in Table 2.

The mater action (46) has the following form:

$$
\begin{aligned}
S= & \int\left(L+\xi \partial_{\mu} \partial_{\nu} h^{\mu \nu}-\partial_{\mu} \xi \partial^{\mu} \xi+h^{*}{ }_{\mu \nu}\left(\partial^{\mu} C^{\nu}\right.\right. \\
& \left.\left.+\partial^{\nu} C^{\mu}\right)-\xi^{*} \partial_{\mu} C^{\mu}\right) d^{4} x .
\end{aligned}
$$

In this expression, the contributions of the resolution degree $0,1,2$ are determined by formulas (47), (48), (49), with the competition function $\tau$ and structure functions $\Gamma^{\mu \nu}{ }_{\rho}, \Gamma_{\rho}, W$ being defined by the relations (105), (114), (112). All the contributions of resolution degrees vanish identically, $S_{k}=$ $0, k \geq 3$.

The gauge fixing conditions can be introduced in various ways. The first option is to impose the independent gauge fixing conditions (74), which we chose in the form

$\chi^{i}(h)=\partial_{j} h^{i j}-\frac{1}{2} \partial^{i} h^{j}{ }_{j}=0, \quad \xi=0$.

Here, the quantity $h^{i j}, i, j=1,2,3$, denotes the space part of the symmetric tensor $h^{\mu \nu}$. The gauge is not covariant because it is not possible to find the tensor structure with three independent components in four-dimensional Minkowski space.

Given the independent gauge conditions (117), the corresponding Lagrange multipliers, ghosts, and anti-fields read:

$$
\operatorname{gh} \pi_{i}(x)=\operatorname{gh} \pi(x)=0, \quad \operatorname{gh} \bar{C}_{i}(x)=\operatorname{gh} \bar{C}(x)=-1,
$$
$\operatorname{gh} \bar{C}^{* i}(x)=\operatorname{gh} \bar{C}^{*}(x)=0$.

The non-minimal action (76) takes the following form:

$S_{\text {non-min }}=S+\int\left(\bar{C}^{* i} \pi_{i}+\bar{C}^{*} \pi\right) d^{4} x$.

The gauge Fermion (77) reads

$\Psi=\int\left[\bar{C}_{i}\left(\partial_{j} h^{i j}-\frac{1}{2} \partial^{i} h^{j}{ }_{j}\right)+\bar{C} \xi\right] d^{4} x$.

The gauge fixing conditions for anti-fields have the form

$h^{*}{ }_{i j}=-\frac{1}{2}\left(\partial_{i} \bar{C}_{j}+\partial_{j} \bar{C}_{i}-\delta_{i j} \partial_{k} \bar{C}^{k}\right), \quad h^{*}{ }_{0 i}=0, \quad \xi^{*}=\bar{C}$, 


$$
\bar{C}^{* i}=\partial_{j} h^{i j}-\frac{1}{2} \partial^{i} h^{j}{ }_{j}, \quad \bar{C}^{*}=\xi .
$$

The gauge fixed action (79) reads

$$
\begin{aligned}
S_{\Psi}= & \int\left[L+\xi \partial_{\mu} \partial_{\nu} h^{\mu \nu}-\partial_{\mu} \xi \partial^{\mu} \xi+\bar{C}_{i} \Delta C^{i}-\bar{C} \partial_{\mu} C^{\mu}\right. \\
& \left.+\pi_{i}\left(\partial_{j} h^{i j}-\frac{1}{2} \partial^{i} h^{j}{ }_{j}\right)+\pi \xi\right] d^{4} x .
\end{aligned}
$$

The generating functional of Green's functions is determined in the usual way,

$$
\begin{aligned}
Z & =\int[d \varphi] \exp \left(\frac{i}{\hbar} S_{\Psi}\right), \\
\varphi & =\left\{h^{\mu \nu}(x), C^{\mu}(x), \xi(x), \bar{C}_{i}(x), \bar{C}(x), \pi_{i}(x), \pi(x)\right\} .
\end{aligned}
$$

If the compensator field $\xi$ and Lagrange multiplier $\pi$ are integrated out of the path integral, we get the following result:

$$
\begin{aligned}
Z= & \int\left[d \varphi^{\prime}\right] \exp \left\{\frac { i } { \hbar } \int \left[L+\bar{C}_{i} \Delta C^{i}-\bar{C} \partial_{\mu} C^{\mu}\right.\right. \\
& \left.\left.+\pi_{i}\left(\partial_{j} h^{i j}-\frac{1}{2} \partial^{i} h^{j}{ }_{j}\right)\right] d^{4} x\right\} \\
\varphi^{\prime}= & \left\{h^{\mu \nu}(x), C^{\mu}(x), \bar{C}_{i}(x), \bar{C}(x), \pi_{i}(x)\right\} .
\end{aligned}
$$

This expression has been previously obtained in the work [8] by means of the modified FP method for the theories with unfree gauge symmetry.

Consider another gauge fixing. Introduce covariant gauge fixing condition of the following form:

$\chi^{\mu}(h)=\partial_{\nu} h^{\mu \mu}=0, \quad \xi=0$.

This gauge is redundant because the gauge conditions are not independent on the mass shell,

$\partial_{\mu} \chi^{\mu}=\partial_{\mu} \partial_{\nu} h^{\mu \mu} \approx 0$

The operator of identity (81) is just a divergence,

$\Delta_{\alpha}^{a} \equiv \Delta_{\mu}=\partial_{\mu}$

The null vectors of $\Delta_{\mu}$ are irreducible in the sence (82), with $\Lambda$ being chosen as follows:

$\Lambda_{a}^{\alpha} \equiv \Lambda^{\mu}=\partial^{\mu}$

In this case, $M(82)$ is the D'Alembert operator,

$$
\Lambda^{\mu} \Delta_{\mu}=\square, \quad \operatorname{det} \square \neq 0 .
$$

The set of fields and anti-fields (83), (84) of non-minimal sector read

$$
\begin{aligned}
& \operatorname{gh} \bar{C}_{\mu}(x)=\operatorname{gh} \bar{C}(x)=\operatorname{gh} \zeta^{*}(x)=\operatorname{gh} \pi^{* \mu}(x) \\
& =\operatorname{gh} \pi^{*}(x)=-\operatorname{gh} \lambda(x)=-1 ; \quad \operatorname{gh} \lambda^{*}(x)=-2 ; \\
& \operatorname{gh} \zeta(x)=\operatorname{gh} \pi_{\mu}(x)=\operatorname{gh} \bar{C}^{* \mu}(x)=\operatorname{gh} \bar{C}^{*}(x)=0
\end{aligned}
$$

The associated non-minimal master-action (85) has the form,

$S_{n o m-\min }=S+\int\left(\bar{C}^{* \mu} \pi_{\mu}+\bar{C}^{*} \pi+\zeta^{*} \lambda\right) d^{4} x$

The gauge Fermion (86) reads

$\Psi=\int\left(\bar{C}_{\mu}\left(\partial_{\nu} h^{\mu \nu}+\partial^{\mu} \zeta\right)+\bar{C} \xi\right) d^{4} x$.

The anti-fields express as follows:

$$
\begin{aligned}
h_{\mu \nu}^{*} & =-\frac{1}{2}\left(\partial_{\mu} \bar{C}_{\nu}+\partial_{\nu} \bar{C}_{\mu}\right), \quad \xi^{*}=\bar{C}, \\
\bar{C}^{* \mu} & =\partial_{\nu} h^{\nu \mu}+\partial^{\mu} \zeta, \\
\bar{C}^{*} & =\xi, \quad \zeta^{*}=-\partial^{\nu} \bar{C}_{\nu} .
\end{aligned}
$$

For the gauge fixed action (87), we get

$$
\begin{aligned}
S_{\Psi}= & \int\left[L+\xi \partial_{\mu} \partial_{\nu} h^{\mu \nu}-\partial_{\mu} \xi \partial^{\mu} \xi-\partial_{\mu} \bar{C}_{\nu}\left(\partial^{\mu} C^{\mu}\right.\right. \\
& \left.+\partial^{\mu} C^{v}\right)-\bar{C} \partial_{\mu} C^{\mu} \\
& \left.+\pi_{\mu}\left(\partial_{\nu} h^{\mu \nu}+\partial^{\mu} \zeta\right)+\pi \xi-\partial^{\mu} \bar{C}_{\mu} \lambda\right] d^{4} x
\end{aligned}
$$

The generating functional of Green's functions read

$$
\begin{aligned}
Z & =\int[d \varphi] \exp \left(\frac{i}{\hbar} S_{\Psi}\right), \\
\varphi & =\left\{h^{\mu \nu}(x), C^{\mu}(x), \xi(x), \bar{C}_{\mu}(x), \bar{C}(x), \pi_{\mu}(x), \pi(x), \zeta(x), \lambda(x)\right\}
\end{aligned}
$$

Let us see that the expressions (123) and (135) define one and same quantity $Z$. To make explicit comparison of results with independent and redundant gauge, we bring (135) to the form (124). We proceed with making the change of ghost variables,

$$
\begin{aligned}
\bar{C}_{0} & =\bar{C}^{\prime}{ }_{0}-\partial_{0} \partial^{i} C^{\prime}{ }_{i}, \quad \bar{C}_{i}=\partial_{0} \partial^{0} \bar{C}^{\prime}{ }_{i}, \\
\pi_{0} & =\pi^{\prime}{ }_{0}-\partial_{0} \partial^{i} \pi^{\prime}{ }_{i}, \quad \pi_{i}=\partial_{0} \partial^{0} \pi^{\prime}{ }_{i}, \\
\partial_{0} \zeta & =\zeta^{\prime}, \quad \partial_{0} \lambda=\lambda^{\prime} .
\end{aligned}
$$

This change of the variables preserves the integration measure because the variables of opposite Grassmann parity transform with one and the same transformation law. The intermediate result for the path integral reads 


$$
\begin{aligned}
Z= & \int\left[d \varphi^{\prime}\right] \exp \left(\frac { i } { \hbar } \int \left[L+\xi \partial_{\mu} \partial_{\nu} h^{\mu \nu}-\partial_{\mu} \xi \partial^{\mu} \xi+\bar{C}^{\prime}{ }_{i}\left(\partial_{0} \partial^{0} \square C^{i}+\partial_{0} \partial^{i} \square C^{0}\right)-\bar{C} \partial_{\mu} C^{\mu}\right.\right. \\
& \left.\left.+\bar{C}^{\prime}{ }_{0}\left(\partial_{\mu} \partial^{\mu} C^{0}+\partial^{0} \partial_{\mu} C^{\mu}\right)+\pi^{\prime}{ }_{i}\left(\partial_{0} \partial^{0} \partial_{\mu} h^{\mu i}-\partial^{i} \partial_{0} \partial_{\mu} h^{\mu 0}\right)+\pi^{\prime}{ }_{0}\left(\zeta^{\prime}+\partial_{\mu} h^{\mu 0}\right)+\bar{C}^{\prime}{ }_{0} \lambda^{\prime}+\pi \xi\right] d^{4} x\right), \\
& \varphi^{\prime}=\left\{h^{\mu \nu}(x), \xi(x), C^{\mu}(x), \bar{C}^{\prime}{ }_{\mu}(x), \bar{C}(x), \pi^{\prime}{ }_{\mu}(x), \pi(x), \zeta^{\prime}(x), \lambda^{\prime}(x)\right\} .
\end{aligned}
$$

In this expression, the variables $\zeta^{\prime}, \lambda^{\prime}, \pi^{\prime}{ }_{0}, \bar{C}^{\prime}{ }_{0}$ can be integrated out,

$$
\begin{aligned}
& \int\left[d \pi^{\prime}{ }_{0}\right]\left[d \bar{C}^{\prime}{ }_{0}\right]\left[d \zeta^{\prime}\right]\left[d \lambda^{\prime}\right] \exp \left(\frac { i } { \hbar } \int \left[\pi^{\prime}{ }_{0}\left(\zeta^{\prime}+\partial_{\mu} h^{\mu 0}\right)\right.\right. \\
& \left.\left.+\bar{C}^{\prime}{ }_{0}\left(\lambda^{\prime}+\partial_{\mu} \partial^{\mu} C^{0}+\partial^{0} \partial_{\mu} C^{\mu}\right)\right] d^{4} x\right) \\
& =\int\left[d \pi^{\prime}{ }_{0}\right]\left[d \bar{C}^{\prime}{ }_{0}\right] \delta\left(\pi^{\prime}{ }_{0}\right) \delta\left(\bar{C}^{\prime}{ }_{0}\right) \exp \left(\frac { i } { \hbar } \int \left[\pi^{\prime}{ }_{0} \partial_{\mu} h^{\mu 0}\right.\right. \\
& \left.\left.+\bar{C}^{\prime}{ }_{0}\left(\partial_{\mu} \partial^{\mu} C^{0}+\partial^{0} \partial_{\mu} C^{\mu}\right)\right] d^{4} x\right)=\text { const. }
\end{aligned}
$$

After simplifications, we get

$$
\begin{aligned}
Z= & \int\left[d \varphi^{\prime \prime}\right] \exp \left(\frac { i } { \hbar } \int \left[L+\xi \partial_{\mu} \partial_{\nu} h^{\mu \nu}-\partial_{\mu} \xi \partial^{\mu} \xi\right.\right. \\
& +\bar{C}^{\prime}{ }_{i}\left(\partial_{0} \partial^{0} \square C^{i}+\partial_{0} \partial^{i} \square C^{0}\right)-\bar{C} \partial_{\mu} C^{\mu} \\
& \left.\left.+\pi^{\prime}{ }_{i}\left(\partial_{0} \partial^{0} \partial_{\mu} h^{\mu i}-\partial^{i} \partial_{0} \partial_{\mu} h^{\mu 0}\right)+\pi \xi\right] d^{4} x\right), \\
\varphi^{\prime \prime}= & \left\{h^{\mu v}(x), \xi, C^{\mu}(x), \bar{C}^{\prime}{ }_{i}(x), \bar{C}(x), \pi^{\prime}{ }_{i}(x), \pi(x)\right\} .
\end{aligned}
$$

This expression can be obtained from the gauge fixed action (79) in the independent gauge,

$\chi^{\prime i}(h)=\partial_{0} \partial^{0} \partial_{\mu} h^{\mu i}-\partial^{i} \partial_{0} \partial_{\mu} h^{\mu 0}, \quad \xi=0$.

As the path integral is independent of the gauge choice, the expressions (124) and (140) determine one and the same expression for the generating functional of Green's functions of the linearized unimodular gravity.

\section{Concluding remarks}

Let us summarize and discuss the results.

Proceeding from the observation that the reasonable gauge field theories can admit the local quantities termed the completion functions such that vanish on shell and do not reduce to the differential consequences of equations of motion (6), we deduce the most general gauge symmetry algebra for this case. It turns out that the existence of the completion functions in the theory results in the unfree gauge symmetry, with gauge parameters constrained by the equations (24). And vice versa, the unfree gauge symmetry implies the existence of completion functions. This is a consequence of the modified Noether identities (9) which involve both the Lagrangian equations and completion functions. The modified identities result in the unfree gauge algebra. Given the unfree gauge symmetry algebra, we work out a systematic procedure for the BV-BRST embedding of the theory. The extension of the $\mathrm{BV}$ formalism to the systems with unfree gauge symmetry algebra has some distinctions from the case where the gauge parameters are unconstrained. The source of distinctions is two-fold. First, the Koszul-Tate resolution for the ideal of on-shell vanishing local function(al)s $I$ should involve the completion functions as the 1.h.s. of the Lagrangian equations do not generate $I$. Second, the ghost are constrained by the equations (40) as the corresponding gauge parameters are unfree (24). The equations constraining the ghosts (40) have to be also accounted for by the Koszul-Tate resolution. These reasons define the minimal set of the fields and anti-fields needed for the proper BV embedding, see Table 1 in Sect. 3. The set involves the anti-fields $\xi^{a}$ to the constraints imposed on the ghosts (40). What is unusual, these anti-fields have the ghost number zero, while their resolution degree is 1 . The completion functions are also assigned with the anti-fields $\xi_{a}^{*}$. The usual BV formalism with unconstrained gauge symmetry parameters does not involve $\xi, \xi^{*}$. These new variables are naturally conjugate with respect to the anti-bracket. The boundary conditions for the BV masteraction are defined by the original action, gauge generators, completion functions, and operators of gauge parameter constraints (47), (48). In the case of the gauge symmetry with unconstrained gauge parameters, only first two constituents are involved. Given the regularity conditions imposed on the boundary, the master equations admits a solution which is unique modulo the natural ambiguity. The BV formalism for the unfree gauge symmetry admits the re-interpretation in terms of the usual BV formalism for the theory with the "compensator fields", see the remark in the end of Sect. 3. Given the master-action, $\xi$ can be considered on an equal footing with the original gauge fields $\phi$. Then, the theory would correspond to the theory of the fields $\phi, \xi$ with the action $S^{\prime}(\phi, \xi)(70)$ and unconstrained gauge symmetry of the extended set of fields. From the viewpoint of this reinterpretation, the existence theorem of Sect. 5 provides a systematic procedure for inclusion of the compensator fields 
such that the gauge symmetry involves unconstrained parameters of the extended theory. The equivalence is obvious between the original theory and theory with compensators as they correspond to the same BRST complex. In all the examples of specific models with unfree gauge symmetry reviewed in Sect. 6, the compensator fields are known. From the viewpoint of Sect. 5, it looks as an expected fact rather than a coincidence. The reinterpretation of the anti-fields $\xi$ to the equations constraining the ghosts (40) as a compensators can be further extended, in principle, in another direction. The Lagrangian equations can have the lower order differential consequences. This is a typical case for the Lagrangian theories having the second class constraints in the Hamiltonian formalism, for example. Then, Lagrangaian equations do not constitute the involutive PDE system. Concerning the specifics of (non-)involutive equations, see [37]. In particular, the theory admits the implicit Noether identities which involve the original Lagrangian equations and lower order consequences. These consequences and identities control the degree of freedom number on equal footing with the original equations and their gauge symmetries. So, the consistent deformation of the non-involutive Lagrangian theory is not controlled by the naive $\mathrm{BV}$ master equation once it does not respect the consequences and implicit identities [37]. From the algebraic standpoint, the implicit identities are similar to the modified Noether identities (9) if the completion functions $\tau$ are replaced by the lower order differential consequences of the Lagrangian equations. If the BV embedding procedure of Sect. 3 is applied to the non-involutive Lagrangian system, with $\tau_{a}$ being the lower order consequences of the Lagrangian equations, the variables $\xi^{a}$ would play the role of the Stückelberg fields. So, this BV embedding algorithm would work as a systematic procedure of consistent inclusion of Stückelberg fields. In terms of Hamiltonian formalism, the general methods are known of conversion the second class constraints into the first class ones, [20,21], while at Lagrangian level the "Stückelbergization" is rather a series of ad hoc tricks adjusted to specific models than a general systematic method. We expect that the BV embedding procedure of Sect. 3 can be reshaped into the general method of "Stückelbergization" in the Lagrangian formalism. One more aspect of the general connection between the theory with unfree gauge symmetry (hence, with nontrivial completion functions) and its equivalent with the compensator fields and without constraints on the gauge parameters is related to the "global modes". As one can see from the examples, the completion functions usually reduce to the arbitrary constants on shell (see in Sect. 6). With the fixed boundary conditions imposed on the fields, these constants take fixed values defined by the boundary. If the setup is adopted with unfixed boundary conditions for the fields, these constants would play the role of the global conserved quantities. The corresponding global degrees of freedom can be understood as modular parameters. These degrees of freedom have to be accounted for in the BV formalism once the fields are not fixed at the boundary. This issue is not addressed in the present work, though the formalism can accommodate the modular parameters, in principle.

Acknowledgements We thank A. Sharapov for discussions. The work is partially supported by Tomsk State University Competitiveness Improvement Program. The work of SLL is supported by the project 3.5204.2017/6.7 of Russian Ministry of Science and Education.

Data Availability Statement This manuscript has no associated data or the data will not be deposited. [Authors' comment: The paper contains formal developments in theoretical physics. There are no data associated to them.]

Open Access This article is distributed under the terms of the Creative Commons Attribution 4.0 International License (http://creativecomm ons.org/licenses/by/4.0/), which permits unrestricted use, distribution, and reproduction in any medium, provided you give appropriate credit to the original author(s) and the source, provide a link to the Creative Commons license, and indicate if changes were made. Funded by SCOAP S $^{3}$

\section{References}

1. I.A. Batalin, G.A. Vilkovisky, Gauge algebra and quantization. Phys. Lett. 102B, 27 (1981)

2. I.A. Batalin, G.A. Vilkovisky, Feynman rules for reducible gauge theories. Phys. Lett. 120B, 166 (1983)

3. I.A. Batalin, G.A. Vilkovisky, Quantization of gauge theories with linearly dependent generators. Phys. Rev. D 28, 2567-2582 (1983)

4. I.A. Batalin, G.A. Vilkovisky, Existence theorem for gauge algebra. J. Math. Phys. 26, 172-184 (1985)

5. M. Henneaux, C. Teitelboim, Quantization of gauge systems (University of Princeton, Princeton, 1992), p. 520

6. S.L. Lyakhovich, A.A. Sharapov, BRST theory without Hamiltonian and Lagrangian. JHEP 0503, 011 (2005). arXiv:hep-th/0411247

7. P.O. Kazinski, S.L. Lyakhovich, A.A. Sharapov, Lagrange structure and quantization. JHEP 0507, 076 (2005). arXiv:hep-th/0506093

8. D.S. Kaparulin, S.L. Lyakhovich, A note on unfree gauge symmetry. arXiv:1904.04038 [hep-th]

9. R. de Leon Ardon, N. Ohta, R. Percacci, The path integral of unimodular gravity. Phys. Rev. D 97, 026007 (2018). arXiv: 1710.02457 [gr-qc]

10. R. Percacci, Unimodular quantum gravity and the cosmological constant. Found. Phys. 48, 1364-1379 (2018)

11. B.L. Voronov, I.V. Tyutin, Formulation of gauge theories of general form. I. Theor. Math. Phys 50, 218 (1982)

12. B.L. Voronov, I.V. Tyutin, [Teor. Mat. Fiz. 50 (1982) 333]

13. J.M.L. Fisch, M. Henneaux, Homological perturbation theory and the algebraic structure of the antifield: antibracket formalism for gauge theories. Commun. Math. Phys. 128, 627 (1990)

14. J.M.L. Fisch, M. Henneaux, J. Stasheff, C. Teitelboim, Existence, uniqueness and cohomology of the classical BRST charge with ghosts of ghosts. Commun. Math. Phys. 120, 379 (1989)

15. B.S. DeWitt, Dynamical theory of groups and fields. Conf. Proc. C 585 (1964) 630701 [Les Houches Lect. Notes 13 (1964) 585]

16. A. Maznytsia, C.R. Preitschopf, D.P. Sorokin, Duality of selfdual actions. Nucl. Phys. B 539, 438 (1999). arXiv:hep-th/9805110 
17. X. Bekaert, M. Henneaux, Comments on chiral p forms. Int. J. Theor. Phys. 38, 1161 (1999). arXiv:hep-th/9806062

18. I. Bandos, On Lagrangian approach to self-dual gauge fields in spacetime of nontrivial topology. JHEP 1408, 048 (2014). arXiv:1406.5185 [hep-th]

19. D. Francia, S.L. Lyakhovich, A.A. Sharapov, On the gauge symmetries of Maxwell-like higher-spin Lagrangians. Nucl. Phys. B 881, 248 (2014). arXiv: 1310.8589 [hep-th]

20. I.A. Batalin, I.V. Tyutin, Existence theorem for the effective gauge algebra in the generalized canonical formalism with abelian conversion of second class constraints. Int. J. Mod. Phys. A 6, 3255 (1991)

21. I. Batalin, M. Grigoriev, S. Lyakhovich, Non-abelian conversion and quantization of non-scalar second-class constraints. J. Math. Phys. 46, 072301 (2005). arXiv:hep-th/0501097

22. W. Buchmuller, N. Dragon, Einstein gravity from restricted coordinate invariance. Phys. Lett. B 207, 292-294 (1988)

23. W. Buchmuller, N. Dragon, Gauge fixing and the cosmological constant. Phys. Lett. B 223, 313-317 (1989)

24. W.G. Unruh, A unimodular theory of canonical quantum gravity. Phys. Rev. D 40, 1048 (1989)

25. M. Henneaux, C. Teitelboim, The cosmological constant and general covariance. Phys. Lett. B 222, 195-199 (1989)

26. G.F.R. Ellis, H. van Elst, J. Murugan, J.P. Uzan, On the trace-free einstein equations as a viable alternative to general relativity. Class. Quantum Gravity 28, 225007 (2011). arXiv:1008.1196 [gr-qc]

27. G.F.R. Ellis, The trace-free Einstein equations and inflation. Gen. Relativ. Gravit. 46, 1619 (2014). arXiv:1306.3021 [gr-qc]
28. A. Padilla, I.D. Saltas, A note on classical and quantum unimodular gravity. Eur. Phys. J. C 75(11), 561 (2015). arXiv:1409.3573 [grqc]

29. S. Gielen, R. de León Ardón, R. Percacci, Gravity with more or less gauging. Class. Quantum Gravity 35(19), 195009 (2018). arXiv: 1805.11626 [gr-qc]

30. A.O. Barvinsky, A.Y. Kamenshchik, Darkness without dark matter and energy: generalized unimodular gravity. Phys. Lett. B 774, 59 (2017). arXiv:1705.09470 [gr-qc]

31. A.O. Barvinsky, N. Kolganov, A. Kurov, D. Nesterov, Dynamics of the generalized unimodular gravity theory. arXiv:1903.09897 [hep-th]

32. E. Alvarez, D. Blas, J. Garriga, E. Verdaguer, Transverse Fierz-Pauli symmetry. Nucl. Phys. B 756, 148 (2006). arXiv:hep-th/0606019

33. D. Blas, Gauge symmetry and consistent spin-two theories. J. Phys. A 40, 6965 (2007). arXiv:hep-th/0701049

34. E.D. Skvortsov, M.A. Vasiliev, Transverse invariant higher spin fields. Phys. Lett. B 664, 301 (2008). arXiv:hep-th/0701278 [HEP$\mathrm{TH}]$

35. A. Campoleoni, D. Francia, Maxwell-like Lagrangians for higher spins. JHEP 1303, 168 (2013). arXiv: 1206.5877 [hep-th]

36. D. Francia, G.L. Monaco, K. Mkrtchyan, Cubic interactions of Maxwell-like higher spins. JHEP 1704, 068 (2017). arXiv:1611.00292 [hep-th]

37. D.S. Kaparulin, S.L. Lyakhovich, A.A. Sharapov, JHEP 1301, 097 (2013). https://doi.org/10.1007/JHEP01(2013)097. arXiv:1210.6821 [hep-th] 